Full length article

\title{
Remote triggering of TGF- $\beta /$ Smad2/3 signaling in human adipose stem cells laden on magnetic scaffolds synergistically promotes tenogenic commitment
}

\author{
Ana M. Matoss a,b, Ana I. Gonçalves a,b,*, Márcia T. Rodrigues ${ }^{a, b}$, Margarida S. Miranda ${ }^{a, b}$, \\ Alicia J. El Haj ${ }^{c}$, Rui L. Reis ${ }^{\mathrm{a}, \mathrm{b}}$, Manuela E. Gomes ${ }^{\mathrm{a}, \mathrm{b}, *}$ \\ a 3B's Research Group, I3Bs - Research Institute on Biomaterials, Biodegradables and Biomimetics, University of Minho, Headquarters of the European \\ Institute of Excellence on Tissue Engineering and Regenerative Medicine, Avepark - Zona Industrial da Gandra, 4805-017 Barco, Guimarães, Portugal \\ ${ }^{\mathrm{b}}$ ICVS/3B's - PT Government Associate Laboratory, Braga, Guimarães, Portugal \\ ${ }^{\mathrm{c}}$ Healthcare Technologies Institute, Birmingham University, B15 2TT, Birmingham, United Kingdom
}

\section{A R T I C L E I N F O}

\section{Article history:}

Received 17 January 2020

Revised 1 July 2020

Accepted 2 July 2020

Available online 8 July 2020

\section{Keywords:}

TGF- $\beta /$ Smad $2 / 3$ signaling pathway

Tenogenic differentiation

Magnetic scaffolds

Magnetic stimulation

\begin{abstract}
A B S T R A C T
Injuries affecting load bearing tendon tissues are a significant clinical burden and efficient treatments are still unmet. Tackling tendon regeneration, tissue engineering strategies aim to develop functional substitutes that recreate native tendon milieu. Tendon mimetic scaffolds capable of remote magnetic responsiveness and functionalized magnetic nanoparticles (MNPs) targeting cellular mechanosensitive receptors are potential instructive tools to mediate mechanotransduction in guiding tenogenic responses. In this work, we combine magnetically responsive scaffolds and targeted Activin A type II receptor in human adipose stem cells (hASCs), under alternating magnetic field (AMF), to synergistically facilitate external control over signal transduction. The combination of remote triggering TGF- $\beta / \operatorname{Smad} 2 / 3$ using MNPs tagged hASCs, through magnetically actuated scaffolds, stimulates overall expression of tendon related genes and the deposition of tendon related proteins, in comparison to non-stimulated conditions. Moreover, the phosphorylation of Smad2/3 proteins and their nuclear co-localization was also more evident. Overall, biophysical stimuli resulting from magnetic scaffolds and magnetically triggered cells under AMF stimulation modulate the mechanosensing response of hASCs towards tenogenesis, holding therapeutic promise.
\end{abstract}

\section{Statement of Significance}

The concept of magnetically-assisted tissue engineering may assist the development of innovative solutions to treat tendon disorders upon remote control of biological processes as cell migration or differentiation. Herein, we originally combine a fibrous aligned superparamagnetic scaffold, based on a biodegrad-

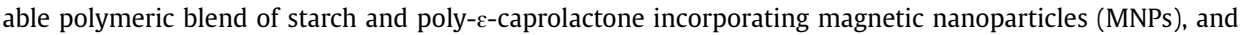
human adipose stem cells (hASCs) labelled with MNPs functionalized with anti-activin receptor type IIA (ActRIIA). Constructs were stimulated using alternating magnetic field (AMF), to activate the ActRIIA and subsequent induction of TGF- $\beta$ signaling, through Smad2/3 phosphorylation cascade, enhancing the expression of tendon-related markers. Altogether, these findings contribute with powerful bio-magnetic approaches to activate key tenogenic pathways, envisioning future translation of magnetic biomaterials into regenerative platforms for tendon repair.

(c) 2020 Acta Materialia Inc. Published by Elsevier Ltd. All rights reserved.

\footnotetext{
* Corresponding authors.

E-mail addresses: ana.goncalves@i3bs.uminho.pt (A.I. Gonçalves), megomes@i3bs.uminho.pt (M.E. Gomes).
} 


\section{Introduction}

Tendons are connective tissues which main function is the transmission of forces between muscles and bones, enabling body motion [1]. Many factors are likely to be involved in the onset of tendon injuries. Intrinsic factors include age, gender, anatomical variants, body weight, systemic disease, and genetic predisposition. Extrinsic factors include sporting activities, physical loading, occupation, and environmental conditions [2]. As a consequence of injury, tendon undergoes a repair process instead of complete regeneration which leads to the formation of a scar tissue limiting tendon gliding and motion amplitude, with a high risk of re-injury and/or associated pain [3]. Currently, classical treatments including oral administration of anti-inflammatory drugs and/or physical rehabilitation and surgical interventions fail in inducing a regenerative process and restoring the original functionality of the tissue [4]. In this context, tissue engineering (TE) emerges as a promising superior option for the development of competent bioartificial substitutes encouraging the regeneration of the damaged tissue. Moreover, magnetically-assisted strategies to remotely deliver stimuli directly to cells is a promising approach in tissue engineering. Magnetic actuation concomitantly combined with magnetic responsive materials represent tools with bioinstructive action in vitro but also upon implantation for remote cell mechanotransduction stimulation.

Previous studies suggest that the actuation of magnetic nanoparticles (MNPs) in response to an external magnetic force within a biomaterial substrate, causes its local deformation [5], which is able to activate/promote cells mechanotransduction mechanisms that will ultimately drive cellular responses [6,7]. Strategies applying magnetic stimuli combined with magnetic responsive scaffolds have shown positive outcomes in bone [8], cardiac [9], and vascular [10] TE. Our group has recently reported on magnetic responsive fibrous scaffolds for tendon TE, suggesting activation of YAP/TAZ signaling in response to magnetic stimulus [11]. These strategies may have an important role in tenogenic differentiation of stem cells [11-14] and/or in the modulation of the inflammatory response $[15,16]$, envisioning improved repair outcomes. Signaling cascades act as transducers of mechanical forces into downstream mechanosensory molecules. MNPs targeting cell membrane receptors or ion channels, by magnetic mechano activation technology, have been explored as a powerful instructive tool to actuate signaling pathways in human mesenchymal stem cells for TE approaches [13,17-19]. Transforming growth factor (TGF)- $\beta$ signaling, transduced by Smad2/3 cascade, has been most associated to tendon formation, differentiation, and homeostasis [20,21], highlighting its interest as a potential molecular target for magnetic actuated strategies. On the other hand, activin receptor type IIA (ActRIIA) has been suggested to be involved in the regulatory pathway of the mechanosensitive gene Tenomodulin [13,22-24], known as a tendon lineage marker. Upon activation of this receptor, an intracellular signaling through phosphorylation of Smad2/3 is initiated [25].

In a previous work of our group, we reported that targeted ActRIIA in human adipose stem cells (hASCs), using MNPs and magnetic stimulation, induced tenogenic transcriptional responses, through TGF- $\beta / \operatorname{Smad} 2 / 3$ signaling pathway [13]. Moreover, 3D-printed polymeric composites incorporating MNPs within its aligned fiber structure were designed by us showing improved biological performance in comparison with non-magnetic counterparts [12].

Herein we hypothesized that combining the two approaches may potentiate the tenogenic differentiation of hASCs through magnetically assisted tissue engineering tools with magnetic biomaterials serving as mediators of mechanotransduction. Specifically, we propose to assess the synergistic effect of hASCs labelled with anti-ActRIIA functionalized MNPs, seeded onto magnetic scaffolds, and remotely actuated by external magnetic field. The remote actuation of an alternating magnetic field (AMF) was provided by a custom-designed solenoid device, to stimulate cells laden on the magnetic scaffolds and assist the regulation of tendon related markers to drive tenogenesis (Fig. 1).

\section{Materials and methods}

\subsection{Fabrication and characterization of magnetic scaffolds}

\subsubsection{Fabrication of magnetic scaffolds}

The aligned fibrous magnetic scaffolds (magSPCL) were produced by 3D-printing using a biodegradable polymeric blend of starch and polycaprolactone (SPCL, 30/70\%, Novamont) incorporating commercially available iron oxide magnetic nanoparticles (MNPs, 45-00-252, Micromod) in a ratio of 0.018:1 $(\mathrm{w} / \mathrm{w})$ MNPs:SPCL [12]. For this purpose, a SPCL and iron oxide MNPs

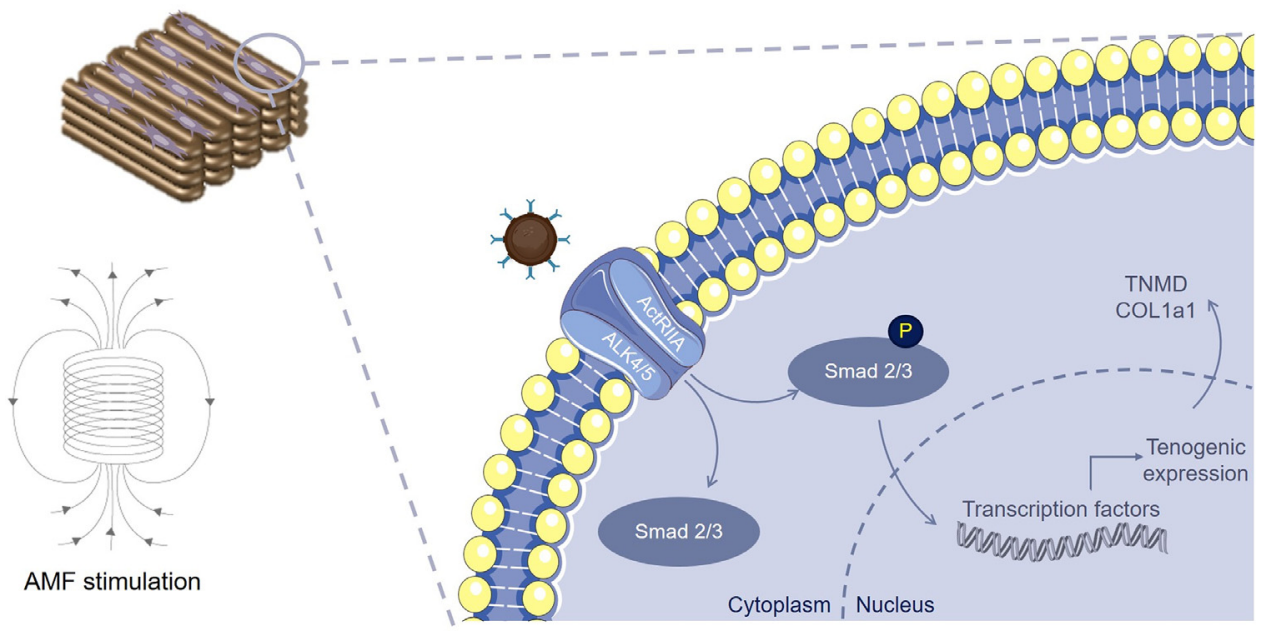

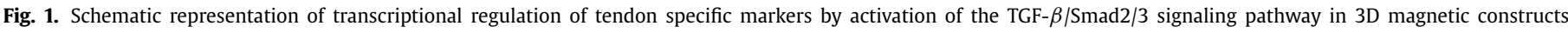

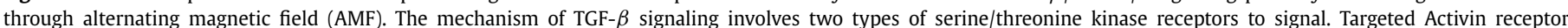

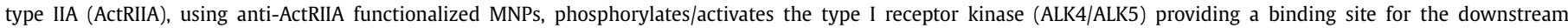

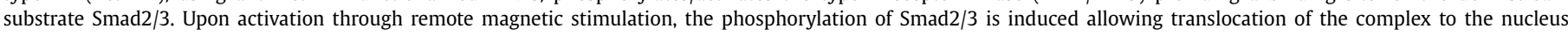
and subsequently inducing tenogenic transcriptional responses. 
mixture was placed in a stainless steel syringe and melted at $120{ }^{\circ} \mathrm{C}$ in a high temperature cartridge unit, then plotted through an 18-gauge needle by air pressure control using a 3D-Bioplotter (4th Generation, Envisiontec). All samples were cut into $4 \times 4 \mathrm{~mm}$ scaffolds and sterilized using ethylene oxide at $42{ }^{\circ} \mathrm{C}$ for $3 \mathrm{~h}$ (EO Gas sterilizer 5XL, $3 \mathrm{M}$ ).

\subsubsection{Determination of weight loss}

The pre-weighed scaffolds were individually immersed in 500 $\mu \mathrm{L}$ of phosphate buffered saline (PBS, $0.01 \mathrm{M}, \mathrm{pH}$ 7.4) solution containing $\alpha$-amylase (A6380, Sigma-Aldrich) at concentration of $145 \mathrm{U} / \mathrm{L}$ [26]. An experimental control of PBS was also performed. All samples were incubated at $37{ }^{\circ} \mathrm{C}$ under constant shaking at $60 \mathrm{rpm}$ for up to 90 days and all solutions were changed twice a week. After each incubation period, a minimum of five samples were removed from the solutions, rinsed in distilled water and let to dry for 3 days at $37^{\circ} \mathrm{C}$. The weight loss was then determined.

\subsubsection{Magnetization analysis}

The magnetic properties of the magSPCL scaffolds were evaluated using a superconducting quantum interface device (SQUIDVSM) magnetometer from Quantum Design under a magnetic field between $-5 \mathrm{~T} \leq B \leq 5 \mathrm{~T}$ at room temperature (RT).

\subsubsection{Micro-Computed tomography analysis and scanning electron microscopy}

The morphology and structure of the magSPCL scaffolds were analysed by high-resolution micro-CT with a Skyscan 1072 scanner (Skyscan, Kontich, Belgium). The cross-section slices from acquired micro-CT angular projections through the scaffolds were reconstructed using the volumetric NRecon reconstruction (version 1.6.6.0, SkyScan) software. CT Vox micro-CT volume rendering software (version 3.3.0) from Bruker was used to build 3D virtual models of the scaffolds from acquired data sets with an original/effective voxel size of $7.535 \mu \mathrm{m}$.

Moreover, structure and morphology of the scaffolds was analyzed by scanning electron microscopy (SEM). After 7 days of culture, magSPCL constructs were rinsed in DPBS and fixed in 10\% formalin (INOPAT) prior to dehydration and coated with gold. Images were collected at an acceleration voltage of $10 \mathrm{kV}$ (JSM-6010LV, JEOL).

\subsection{Functionalization of MNPs and characterization}

\subsubsection{Conjugation of MNPs}

Carboxyl functionalized magnetic nanoparticles (09-02-252, Micromod) were covalently bonded to Anti-Activin receptor type IIA antibody (ab135634, Abcam) (MNPs-ActRIIA) by carbodiimide chemistry as described previously [13] and detailed in the Supplementary Materials and Methods.

Functionalized MNPs (MNPs-ActRIIA) were diluted in $\mathrm{H}_{2} \mathrm{O}$ and analyzed for surface charge and size using a Zetasizer Nano ZS (Malvern Instruments) and NTA (NanoSight, NS, Malvern Instruments) (Supplementary Results). The morphology of the particles before (MNPs) and upon functionalization (MNPs-ActRIIA) were observed by scanning electron microscopy (SEM) (Auriga Compact, Zeiss) analysis. Drops of diluted suspensions were placed in tissue culture coverslips (Sarstedt) and air dried overnight. Prior to analysis, samples were sputter coated with gold, and images were acquired at an acceleration voltage of $5 \mathrm{kV}$. The size, surface charge, and morphology of MNPs-ActRIIA was compared to nonfunctionalized commercial MNPs.

\subsubsection{Fourier transform infrared spectroscopy (FTIR) analysis}

The spectrum of the commercial carboxyl dextran MNPs and of the functionalized MNPs (MNPs-ActRIIA) was obtained by FTIR spectroscopy in attenuated total reflectance mode (FTIR-ATR). For that, a drop of the sample was placed on the crystal. Spectra were obtained at transmittance mode in the range of $4000-400 \mathrm{~cm}^{-1}$ with a resolution of $4 \mathrm{~cm}^{-1}$.

\subsubsection{Pierce BCA protein assay}

To estimate the binding efficiency of the antibody to the MNPs, the supernatants of the particles solutions were collected during the wash steps of the conjugation procedure and analysed by Micro bicinchoninic acid (BCA) assay $(22,235$, Thermo Fisher Scientific), according to manufacturer's instructions. Briefly, $150 \mu \mathrm{L}$ of samples and working reagent were mixed and incubated at $37{ }^{\circ} \mathrm{C}$ for $2 \mathrm{~h}$, and then the absorbance was read at $562 \mathrm{~nm}$. The amount of antibody conjugated to the MNPs was determined according to the difference in protein concentrations between samples. Albumin protein standard solutions and a blank reading of $25 \mathrm{mM}$ glycine were also analysed.

\subsection{Cells isolation and expansion}

Human adipose stem cells (hASCs) were isolated from human liposuction aspirates obtained from healthy females $(n=3)$ with an average age of 42 years, under informed consent, according to the protocols previously approved by the Ethical Committee of Hospital da Prelada (Porto, Portugal). The content of the written informed consent and related procedures were reviewed and approved by the Hospital Ethics Committee. hASCs were isolated from tissue samples and cultured as described before [27,28], and have been previously characterized by RT-PCR for CD44, STRO1, CD105 and CD90 markers, and also for multilineage potential $[27,29]$. Briefly, the tissue was rinsed in phosphate buffered saline (PBS, Sigma-Aldrich) containing $10 \%$ of an Antibiotic/Antimycotic (A/A) solution. The fat solution was immersed in a $0.05 \%$ collagenase type II (Sigma/ C6885) solution for $45 \mathrm{~min}$ at $37{ }^{\circ} \mathrm{C}$ under mild agitation. The digested tissue was centrifuged at $800 \mathrm{~g}$ for $10 \mathrm{~min}$ at $4{ }^{\circ} \mathrm{C}$, after which the supernatant was eliminated. Cells were expanded in basal medium composed of $\alpha$ MEM (12000063, Life Technologies) supplemented with 10\% FBS (15240062, Gibco) and $1 \% \mathrm{~A} / \mathrm{A}$ solution (15240062, Gibco). For all experiments, cells were used at passage 2-4. Three independent experiments were performed, and samples were collected in triplicates.

\subsubsection{Cells labeling with MNPs-ActRIIA and characterization}

hASCs were seeded at a density of 100,000 cells/well in 6-well plates and incubated with MNPs-ActRIIA at $25 \mu \mathrm{g}$ MNPs $/ 2 \times 10^{5}$ cells, for $30 \mathrm{~min}$, in basal serum free medium. After the incubation period, cells were washed with Dulbecco's phosphate-buffered saline (DPBS) (ThermoFisher Scientific) to remove unbound MNPs.

hASCs labeling was confirmed using Prussian Blue (PB) staining, for iron detection, and SEM. For PB assay, cells were stained with a solution of equal parts of $20 \% \mathrm{HCl}$ (30721, Sigma-Aldrich) and $10 \%$ potassium ferrocyanide (P3289, Sigma-Aldrich) for $30 \mathrm{~min}$ before washing 3 times with $\mathrm{dH}_{2} \mathrm{O}$. Then, samples were visualized under a transmitted and reflected light microscope (AxioVert A1 FL LED, Zeiss).

Prior to SEM observation (JSM-6010 LV, JEOL), specimens were dehydrated and coated with gold, and images were collected at an acceleration voltage of $10 \mathrm{kV}$.

\subsection{Assessment of alternating magnetic field parameters effects on hASCs viability and proliferation}

In order to magnetically stimulate the tissue engineered constructs, an alternating magnetic field (AMF) system was developed. This custom designed device consists of a solenoid, in which an alternating electrical current passes through a wire generating a 
uniform magnetic field inside the solenoid (Supplementary Figure S1).

The AMF effect was investigated by varying the frequency $(50 \mathrm{~Hz}$ or $100 \mathrm{~Hz}$ ) to which hASCs were exposed [30]. The effect of AMF actuation in metabolic activity and cell content was investigated at day 0 and day 7 of culture (Supplementary material). The results suggest that the magnetic field resultant from $50 \mathrm{~Hz}$ frequency is promoting higher metabolic activity and cell proliferation after 7 days in culture. Based on this, an AMF with a frequency of $50 \mathrm{~Hz}$ and intensity of $1 \mathrm{mT}$, during $10 \mathrm{~min}$, was selected for the subsequent studies. Non-stimulated groups were kept without magnetic field.

\subsection{Phosphorylation assays of hASCs}

After hASCs labeling with MNPs-ActRIIA, samples were incubated for $10 \mathrm{~min}$ in basal $\alpha$ MEM medium under AMF stimulation $(f=50 \mathrm{~Hz}, B=1 \mathrm{mT})$ or non-stimulated conditions. Cells cultured without MNPs in $\alpha$ MEM medium supplemented with Recombinant Human/Mouse/Rat Activin A Protein (338-AC-010, R\&D systems) were considered positive control of the receptor activation. The dose $(20 \mathrm{ng} / \mathrm{mL})$ and incubation period $(10 \mathrm{~min})$ of Activin A ligand were chosen based on literature and according to the phospho-Smad2/Smad3 ELISA kit [13,31]. Also, to verify the inhibition of the ActRIIA receptor and subsequent cascade of phosphorylation, hASCs were treated for $24 \mathrm{~h}$ with the antagonist SB431542 (72232, Stem cell technologies) $(10 \mu \mathrm{M})$. The TGF- $\beta$ superfamily signal through a receptor complex comprising a type II and type I receptor, both serine/threonine kinases. SB431542 is small-molecule inhibitor of type I activin receptor-like kinase-4, $-5,-7$ (ALK4/ALK5/ALK7), being a selective inhibitor of endogenous activin and TGF- $\beta$ signaling [32]. For the rescue experiments, after pre-treatment with SB431542, cells were incubated with Activin A ligand and MNPs-ActRIIA complex for $10 \mathrm{~min}$ in $\alpha$ MEM medium under AMF stimulation or non-stimulated conditions.

Then, the endogenous levels of phospho-Smad2 (Ser465/467) and phospho-Smad3 (Ser423/425) proteins were quantitatively assessed by ELISA assay (12001, Cell Signaling Technology), following the manufacturer's instructions.

\subsection{Reactive Oxygen Species assay}

The oxidative stress of cells was analysed using a DCFDA - Cellular Reactive Oxygen Species (ROS) Detection Assay (ab113851, Abcam). Briefly, hASCs were seeded at a density of 30,000 cells/well in a dark clear bottom 96-well plate (Corning) and cultured overnight in $\alpha$-MEM medium. The following conditions were assessed: i) hASCs under non-stimulated conditions; ii) hASCs under AMF stimulation; iii) hASCs in $\alpha$-MEM medium supplemented with Activin A (20 ng/mL); iv) hASCs labelled with MNPs-ActRIIA under non-stimulated conditions; or v) hASCs labelled with MNPsActRIIA under AMF stimulation. Cells were stained with DCFDA solution for $45 \mathrm{~min}$ at $37{ }^{\circ} \mathrm{C}$ in the dark and, after that, the fluorescence was read with an excitation of $495 / 20 \mathrm{~nm}$ and an emission of 529/20 nm using a microplate reader (Synergy HT, Bio-TeK Instruments). After this, cells were incubated with TBHP for $4 \mathrm{~h}$ at $37{ }^{\circ} \mathrm{C}$ and the fluorescence was read. hASCs incubated only with DCFDA or incubated with DCFDA and TBHP represent the negative and positive controls, respectively. A blank reading without cells, DCFDA and TBHP was also performed.

\subsection{Culture of hASCs on magSPCL scaffolds}

\subsubsection{Phosphorylation assay on magnetic constructs}

hASCs were seeded onto magSPCL scaffolds at a density of 165,000 cells/scaffold, left to adhere for 1 h30 in $\alpha$-MEM medium, and then the following conditions were assessed: (i) hASCs under non-stimulated conditions; (ii) hASCs under AMF stimulation; (iii) hASCs in $\alpha$-MEM medium supplemented with Activin A (20 ng/mL); (iv) hASCs labelled with MNPs-ActRIIA under nonstimulated conditions; or (v) hASCs labelled with MNPs-ActRIIA under AMF stimulation.

Prior analysis, the constructs were rinsed in ice cold DPBS, scraped in lysis buffer, and sonicated on ice for $10 \mathrm{~min}$. After sonication, the samples were centrifuged and the supernatant stored at $-80{ }^{\circ} \mathrm{C}$. The samples were further analysed by ELISA (PathScan ${ }^{\circledR}$ Phospho-Smad2 (Ser465/467)/Smad3 (Ser423/425), 12,001, Cell Signaling Technology).

\subsubsection{Immunocytochemistry of $\mathrm{pSmad} 2 / 3$}

Cell-laden magSPCL scaffolds were cultured for up to 3 days in basal medium, and AMF stimulation was provided at days 0 and 3. Afterwards, the constructs were rinsed in DPBS and fixed in $10 \%$ formalin (INOPAT) prior to the detection of $\mathrm{pSmad} 2 / 3$ (\#8828, Cell signaling technologies) deposition. After cell permeabilization with $0.1 \%$ Triton-X100 (Sigma/X100)/PBS solution, all samples were blocked using RTU Normal Horse Serum (RTU Vectastain Kit, PK-7200, Vector) for $40 \mathrm{~min}$ at RT, to avoid nonspecific reactions. Then, constructs were incubated overnight with primary antibody against $\mathrm{pSmad} 2 / 3$ diluted in antibody diluent with background reducing components (S3022, Dako) at $4{ }^{\circ} \mathrm{C}$ under gentle agitation. Afterwards, samples were rinsed in $0.1 \%$ Triton-X100 (Sigma/X100)/PBS solution followed by incubation for $1 \mathrm{~h}$ at RT with Alexa fluor 488 secondary antibody (Alfagene). Finally, samples were stained with 4,6-Diamidino-2-phenyindole, dilactate (DAPI, $5 \mu \mathrm{g} / \mu \mathrm{L}$, D9564, Sigma) for $15 \mathrm{~min}$. After washing, the samples were kept in PBS at $4{ }^{\circ} \mathrm{C}$ until further visualization. Immunolabelled samples were acquired by confocal laser scanning microscopy (TCS SP8, Leica) and images analysed using LAX software from Leica.

To assess the co-localization of pSmad $2 / 3$ in the nucleus, Image co-localization measurement was performed to define the overlap between the blue channel (DAPI, nuclei) and the green channel (pSmad2/3 staining) $(n>5)$. Then, area fraction measurement was used to determine the positively stained area. The values were normalized by the total number of nuclei and shown as area of staining (pSmad2/3) per nuclei.

\subsubsection{Long-term culture of hASCs on magSPCL scaffolds}

hASCs were seeded at a density of 65,000 cells/scaffold and cultured for up to 21 days in $\alpha$-MEM medium in the following conditions: i) hASCs under non-stimulated conditions; ii) hASCs under AMF stimulation ( $f=50 \mathrm{~Hz}, B=1 \mathrm{mT}$ ); iii) hASCs in $\alpha$-MEM medium supplemented with Activin A (20 ng/mL); iv) hASCs labelled with MNPs-ActRIIA under non-stimulated conditions; or v) hASCs labelled with MNPs-ActRIIA under AMF stimulation $(f=50 \mathrm{~Hz}, B=1 \mathrm{mT})$. The constructs were further analysed for cell morphology, metabolic activity, proliferation, and iron release by SEM, MTS assay, dsDNA quantification, and ICP, respectively. Moreover, tendon associated markers were assessed by real time RT-PCR, quantification of collagen and non-collagenous proteins, and immunocytochemistry.

2.7.3.1. Iron release measurement. The iron $(\mathrm{Fe})$ released from the magnetic constructs cultured for up to 21 days was evaluated by ICP-OES (Activa M, Horiba Jobin Yvon) using a Burguener MiraMist nebulizer with $1000 \mathrm{~W}$ power. The culture medium was collected after each time-point and frozen at $-80{ }^{\circ} \mathrm{C}$. $\alpha$-MEM medium $(10 \%$ FBS, $1 \% \mathrm{~A} / \mathrm{A}$ ) was used as blank solution for correction purposes. Samples were prepared by adding $250 \mu \mathrm{L}$ of $69 \% \mathrm{HNO}_{3}$ (101799, EMSURE) to $500 \mu \mathrm{L}$ of the samples and then diluted to $5 \% \mathrm{HNO}_{3}$ with ultra-pure $\mathrm{H}_{2} \mathrm{O}$. All samples were filtered with a $0.45 \mu \mathrm{m}$ 
filter (VWR) prior analysis. An iron (Fe,1000 $\mu \mathrm{g} \mathrm{mL}-1$ ) standard solution (13830, Specpure) was used to prepare the diluted standard solutions.

2.7.3.2. MTS assay and DNA quantification. Metabolic activity of hASCs cultured on magSPCL scaffolds was evaluated by MTS assay (Cell Titer 96 Aqueous Solution Cell Proliferation Assay, Promega). Briefly, after 7, 14 and 21 days of culture, the constructs were transferred to another multiwell plate, washed with PBS and incubated for $3 \mathrm{~h}$ at $37{ }^{\circ} \mathrm{C}$ and $5 \% \mathrm{CO}_{2}$ atmosphere in a mixture of serum-free culture medium without phenol red and MTS solution (5:1 ratio). Then, $100 \mu \mathrm{L}$ of each sample was transferred to a 96 well-plate and the absorbance read at $490 \mathrm{~nm}$ (Synergy HT, BioTeK Instruments). The samples were read in triplicate and a blank reading was also performed for correction purposes.

The proliferation of hASCs was determined through DNA quantification of cell lysates, using the Quant-iT ${ }^{\mathrm{TM}}$ PicoGreen ${ }^{\circledR}$ dsDNA kit (P7589, ThermoFisher), after 7, 14 and 21 days of culture. Samples were collected into microtubes with $1 \mathrm{~mL}$ of ultrapure water and stored at $-80{ }^{\circ} \mathrm{C}$. Then, samples were thawed, sonicated, and the supernatant analysed using a microplate reader with an excitation of 485/20 nm and an emission of 528/20 nm (Synergy HT, BioTeK Instruments). Samples and standards were made in triplicate.

2.7.3.3. Real time RT-PCR. Total RNA was extracted using TRI reagent (T9424, Sigma) according to the manufacturer's instructions. Briefly, $600 \mu \mathrm{L}$ of TRI reagent was added to each sample and stored at $-80{ }^{\circ} \mathrm{C}$. After defrosting, samples were incubated with $160 \mu \mathrm{L}$ of chloroform (Sigma) for $15 \mathrm{~min}$ and $12,000 \mathrm{~g}$ centrifuged for $15 \mathrm{~min}$ at $4{ }^{\circ} \mathrm{C}$. The aqueous fraction was collected and
$300 \mu \mathrm{L}$ of isopropanol (Sigma Aldrich) was added. After $10 \mathrm{~min}$, samples were centrifuged at $12,000 \mathrm{~g}$ for $10 \mathrm{~min}$ at $4{ }^{\circ} \mathrm{C}$. RNA pellet was washed with $800 \mu \mathrm{L}$ of $70 \%$ ethanol and subsequently centrifuged at $7500 \mathrm{~g}$ for $5 \mathrm{~min}$ at $4{ }^{\circ} \mathrm{C}$. Air-dried RNA samples were resuspended in $15 \mu \mathrm{L}$ of RNase/DNase free water (Gibco). RNA quantity and purity were determined with a NanoDrop ND-1000 spectrophotometer (NanoDrop Technologies). The cDNA synthesis was performed with the qScript cDNA Synthesis kit (Quanta Biosciences) and using the Mastercycler Realplex (Eppendorf) using an initial amount of total RNA of $1 \mu \mathrm{g}$ in a total volume of $20 \mu \mathrm{L}$. The quantification of the transcripts was carried out by quantitative polymerase chain reaction (qPCR) using the PerfeCTA SYBR Green FastMix kit (Quanta Biosciences) following the manufacturer's protocol, in a Real-Time Mastercycler Realplex thermocycler (Eppendorf). The primers were pre-designed with PerlPrimer v1.1.21 software (Supplementary Table S1) and synthesized by MWG Biotech. $A C T B$ (actin beta) was used as the housekeeping gene. The $2^{-\Delta \Delta C t}$ method was selected to evaluate the relative expression level for each target gene. All values were first normalized against $A C T B$ values, and then to hASCs constructs cultured in basal $\alpha$-MEM medium for 3 days.

2.7.3.4. Quantification of collagen and non-collagenous proteins. The amount of collagen and non-collagenous proteins deposited by hASCs cultured on the magSPCL scaffolds was determined using a semiquantitative assay, namely Sirius Red/Fast Green Collagen Staining Kit (9046, Chondrex). After day 7, day 14 and day 21 of culture, the constructs were fixed with $10 \%$ formalin and stored at $4{ }^{\circ} \mathrm{C}$ until analysis. Briefly, the dye solution was added and incubated for $40 \mathrm{~min}$ in order to completely immerse the fixed cells.
A

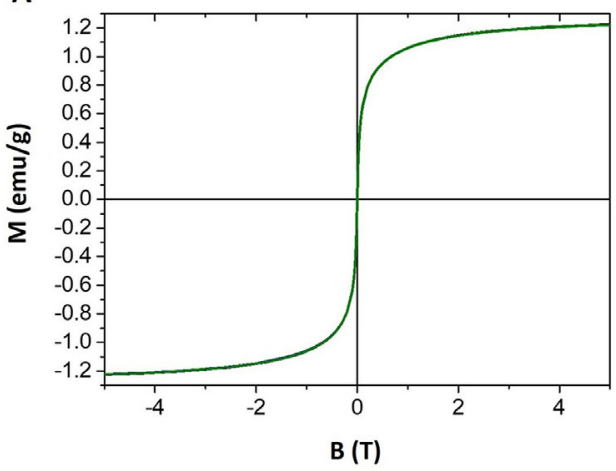

B

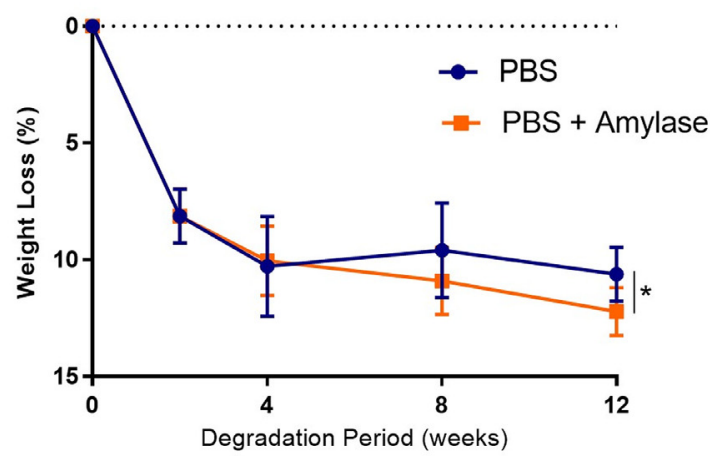

C

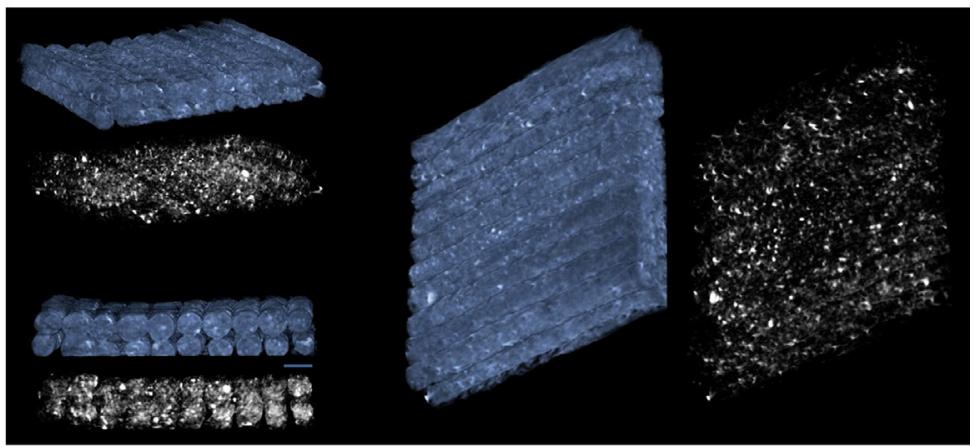

D

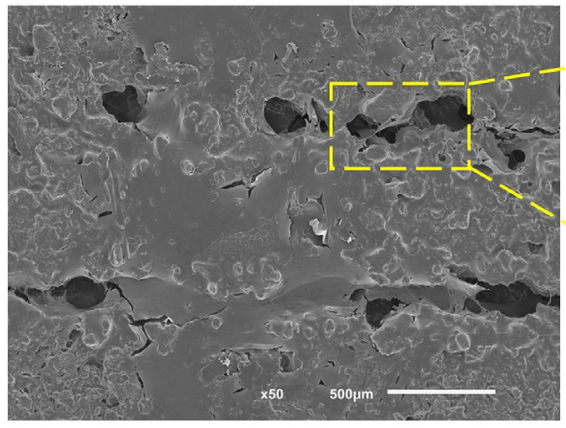

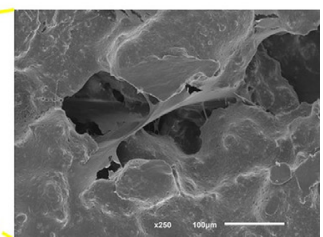

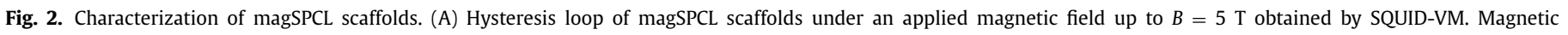

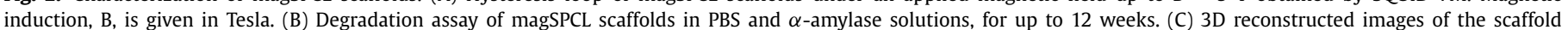

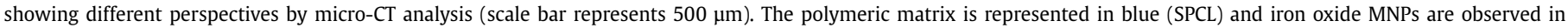

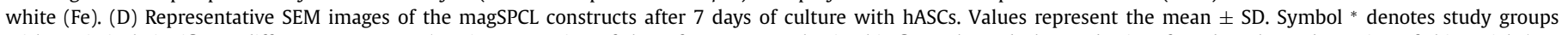
with statistical significant difference $p<0.05$. (For interpretation of the references to color in this figure legend, the reader is referred to the web version of this article.) 
A dye extraction buffer was then mixed and the OD values read in a spectrophotometer (Synergy HT, Biotek Instruments) at $540 \mathrm{~nm}$ and $605 \mathrm{~nm}$.

2.7.3.5. Immunocytochemistry. Cell-laden magSPCL scaffolds were rinsed in DPBS and fixed in $10 \%$ formalin (INOPAT) prior to the detection of Scleraxis (ab58655, Abcam) and Tenomodulin (ab81328, Abcam) deposition, according to the procedure described above for $\mathrm{pSmad} 2 / 3 \mathrm{immunolocation.} \mathrm{After} \mathrm{that,} \mathrm{the} \mathrm{samples} \mathrm{were}$ kept in PBS at $4{ }^{\circ} \mathrm{C}$ until further visualization. Immunolabelled samples were acquired by confocal laser scanning microscopy (TCS SP8, Leica) and images processed using LAX software from Leica.

Semi-quantification of immunocytochemistry was performed using ImageJ by measuring the mean fluorescence intensity of microscopy images ( $n>5)$ acquired in different regions of the scaffold. Briefly, images were thresholded to separate the signal from the background using Huang method [33,34], and then the mean fluorescence values were determined and normalized by the total signal area.

\subsection{Statistical analysis}

Quantitative results are expressed as the mean \pm standard deviation. Statistical analysis of data was performed using GraphPad PRISM version 6.01. The unpaired t-test was performed to compare two normal distributed populations. One-way analysis of variance (ANOVA) followed by the Tukey post hoc test for multiple comparisons or two-way analysis of variance (ANOVA) followed by the Holm-Sidak test for multiple comparisons was performed to compare groups of variables. Differences were considered significant when the $p$ value was $<0.05$.

\section{Results and discussion}

\subsection{Production and characterization of magSPCL scaffolds}

Aligned fibrous magnetic scaffolds (magSPCL) were successfully produced by 3D-printing using a blend of starch and polycaprolactone incorporating iron oxide magnetic nanoparticles, as previously reported by us [12].
A

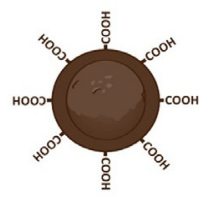

Dextran coated MNPs

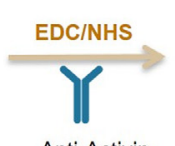

Anti-Activin

Receptor

Type IIA antibody

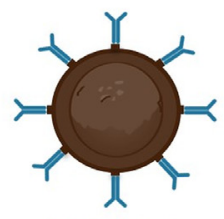

MNPs-ActRIIA

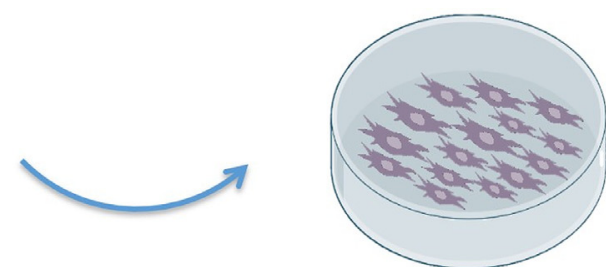

hASCs labelling with MNPs-ActRIIA

B

Dynamic Light Scatering

\begin{tabular}{ccc}
\hline Particles & Size $(\mathbf{n m})$ & $\begin{array}{c}\text { Zeta Potential } \\
(\mathbf{m V})\end{array}$ \\
\hline MNPs & $272 \pm 1.7$ & $-24.9 \pm 0.87$ \\
MNPs - ActRIIA & $310 \pm 3.02$ & $-16 \pm 1.33$ \\
\hline
\end{tabular}

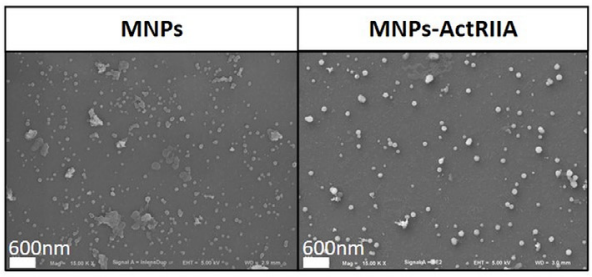

C

\begin{tabular}{|c|c|c|c|}
\hline \multicolumn{4}{|c|}{ Micro BCA Protein Assay Kit } \\
\hline \multirow{2}{*}{ Samples } & \multicolumn{2}{|c|}{$\begin{array}{l}\text { Supernatant protein concentration } \\
\qquad(\mu \mathrm{g} / \mathrm{mL})\end{array}$} & \multirow{2}{*}{$\begin{array}{l}\text { Efficiency } \\
\text { (\% protein on } \\
\text { MNPs) }\end{array}$} \\
\hline & pre coating & post coating & \\
\hline MNPs - ActRIIA & 100 & $63 \pm 13$ & $37 \pm 13$ \\
\hline
\end{tabular}

FTIR spectroscopy

D
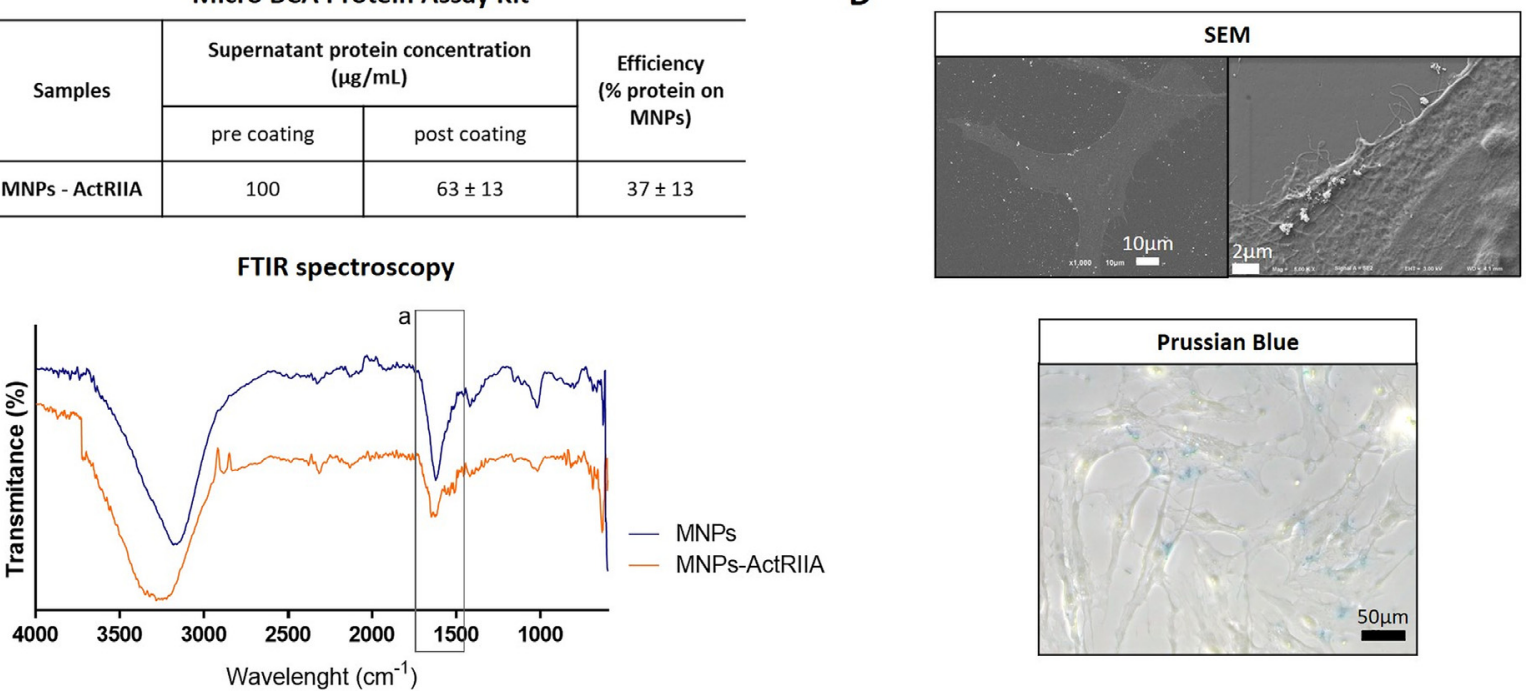

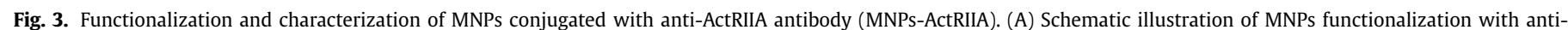

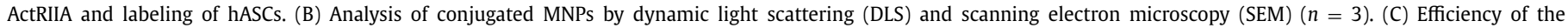

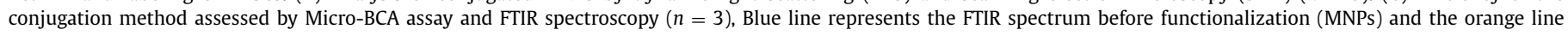
represents the FTIR spectrum after functionalization (MNPs-ActRIIA). (D) SEM and Prussian Blue staining of MNPs-ActRIIA labelled hASCs $(n=3$ ). 
The SQUID-VM analysis of the magSPCL scaffolds in which the magnetization ( $\mathrm{M})$ as a function of the applied magnetic field (B) (hysteresis loop) (Fig. 2A) revealed the absence of the coercive forces and remanence magnetization at zero magnetic field. This fact, confirms the superparamagnetic behavior of the magnetic scaffolds. Furthermore, a saturation magnetization (Ms) value

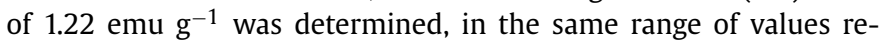
ported for other developed magnetic scaffolds [11,12,35].

The material weight loss (\%) was assessed by comparing the final weight of the material after different periods (2, 4, 8 and 12 weeks) in degradation solutions, with the initial weight (Fig. 2B). For samples immersed in PBS solution, a weight loss of $10.62 \% \pm$ 1.03 was observed while in the presence of $\alpha$-amylase, magSPCL scaffolds present a weight loss of $12.2 \% \pm 0.93$ which demonstrates that the presence of the enzyme increases the degradation rate of the scaffolds. The same degradation behavior was observed in previous studies for SPCL scaffolds [26,36,37]. Also, the solutions collected during the degradation assay were analysed by ICP-OES (Supplementary material), and the iron concentration was found to be below $50 \mu \mathrm{g} \mathrm{L}^{-1}$ in all the degradation solutions (quantification limit of the method $50 \mu \mathrm{g} \mathrm{L}^{-1}$ ).

A

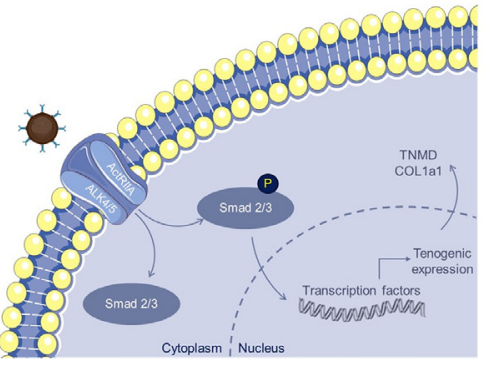

B 2

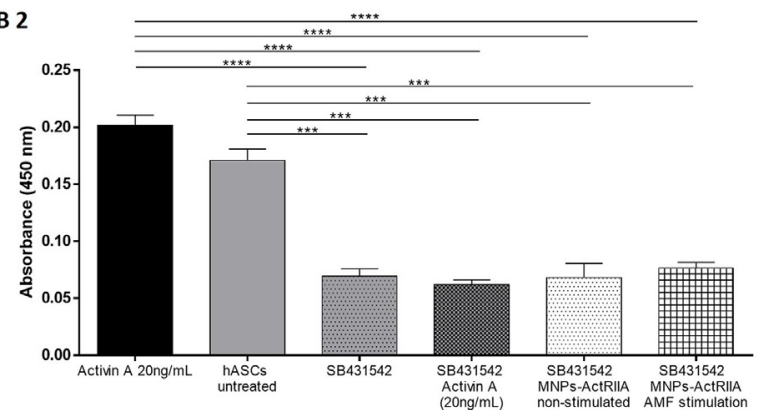

The alignment of the fibers and the effectiveness of MNPs incorporation in the 3D structure was confirmed by micro-CT analysis (Fig. 2C), showing the parallel alignment of the polymeric fibers and the presence of MNPs homogeneously distributed in the fiber network. Moreover, fiber diameter measurements (657.48 \pm 49.48 ) $\mu \mathrm{m}$ were assessed by microscopy images acquired in different regions of the scaffold $(n>10)$.

In Fig. 2D, representative SEM images of the magSPCL constructs after 7 days of culture further support the microarchitectural alignment of the scaffolds, showing the fibers alignment, grooves distribution, and cellular bridging between fibers.

\subsection{Development and characterization of functionalized MNPS}

After functionalization of MNPs with anti-ActRIIA antibody by carbodiimide chemistry (Fig. 3A), the size and surface charge of MNPs and MNPs-ActRIIA were determined by DLS (Fig. 3B) and NTA (Supplementary Figure S2). Results show that the particles size increased after anti-ActRIIA conjugation which confirms the functionalization of the MNPs with the antibody. Furthermore, the surface charge increased in MNPs-ActRIIA suggesting an improved

B 1

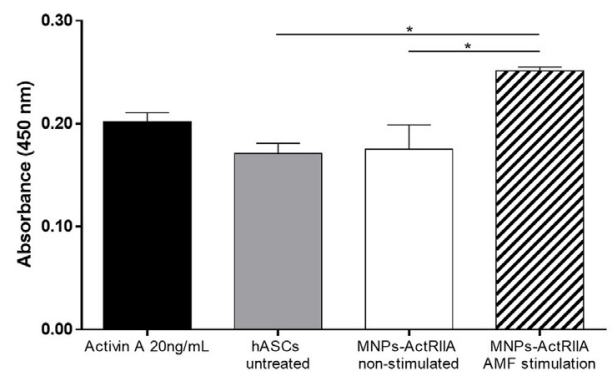

D1

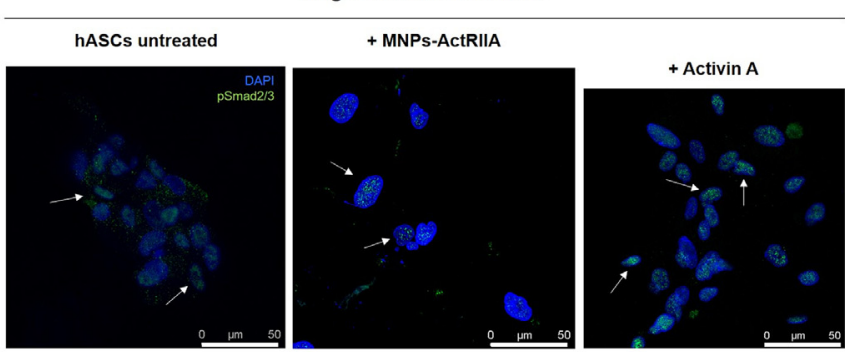

C

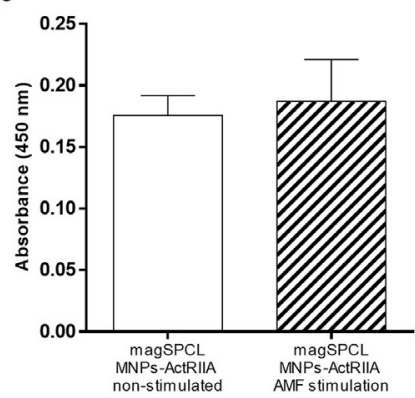

D2

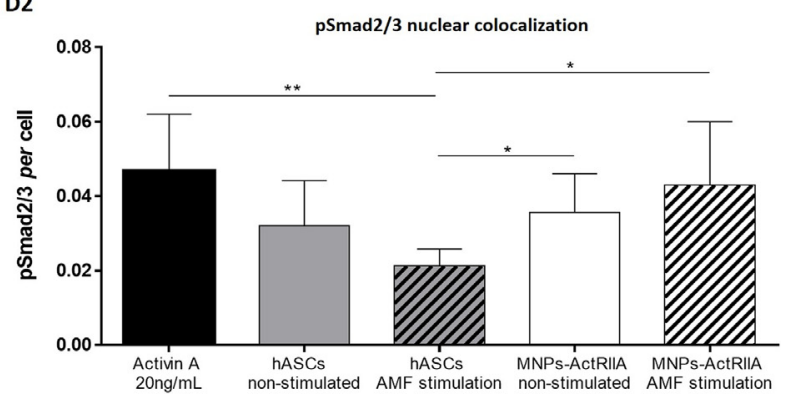

magSPCL AMF stimulation

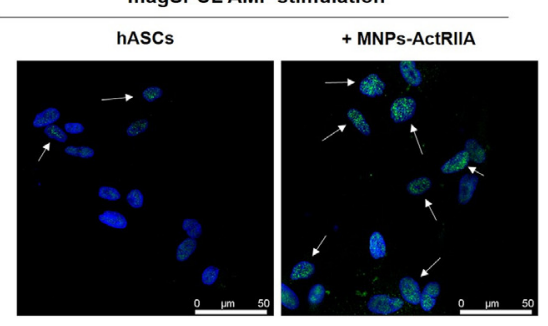

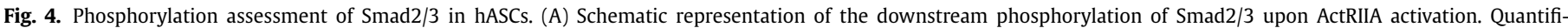

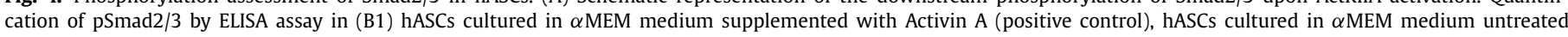

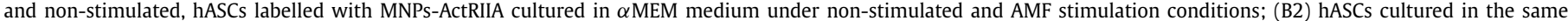

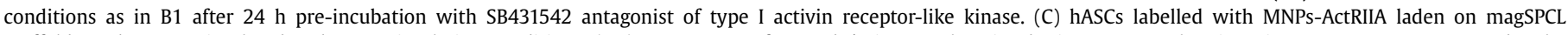

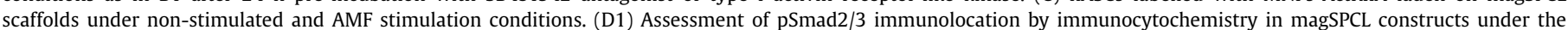

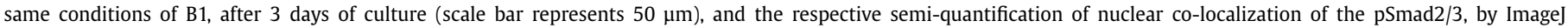

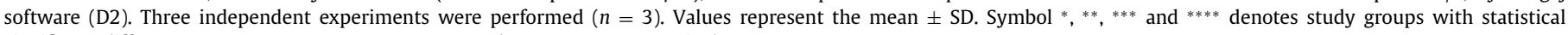
significant difference $p<0.05, p<0.01, p<0.001$ and $p<0.0001$, respectively. 
A

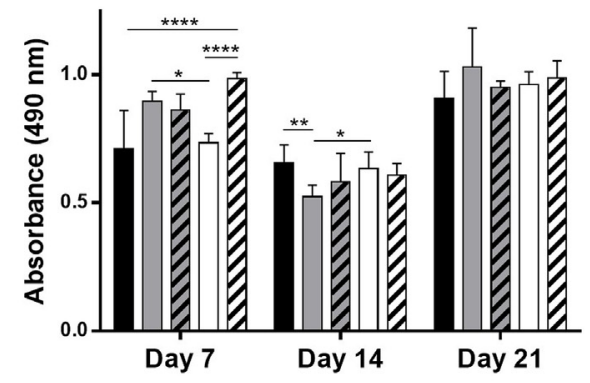

B

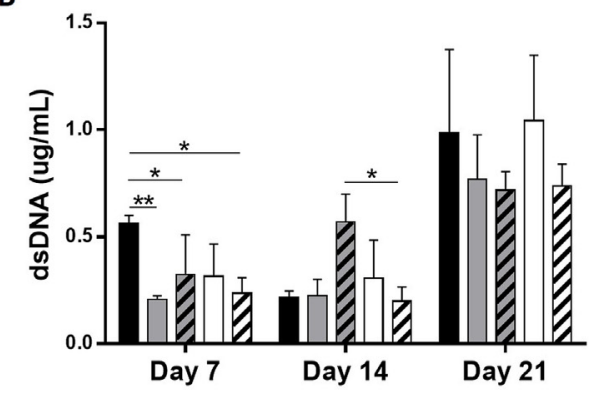

Activin A 20ng/mL

hASCs non-stimulated $\square$ MNPs-ActRIIA non-stimulated

WA hASCs AMF stimulation $\mathbf{Z 7}$ MNPs-ActRIIA AMF stimulation
C

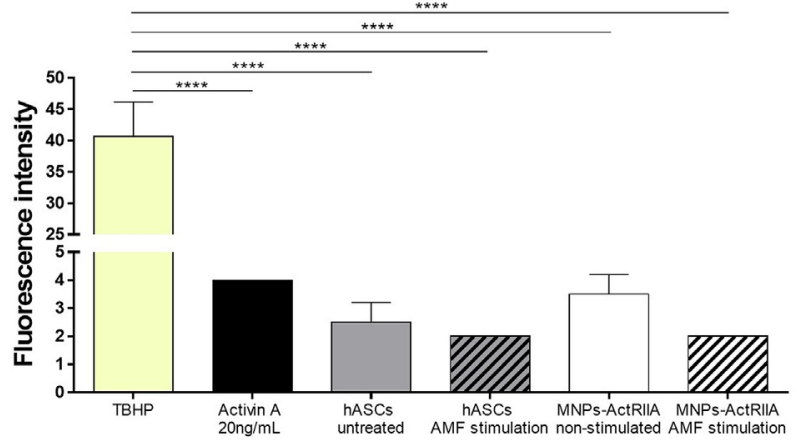

D

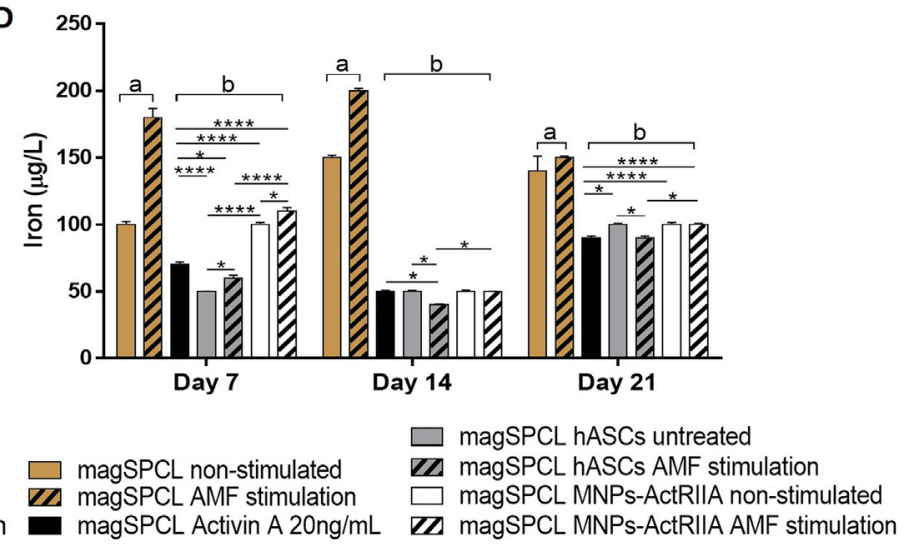

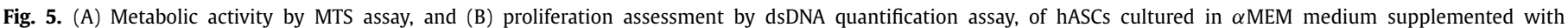

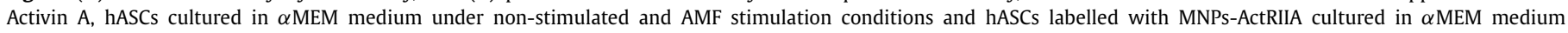

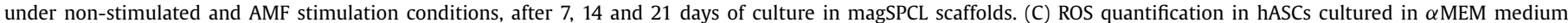

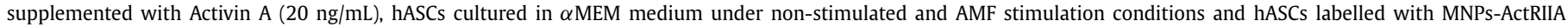

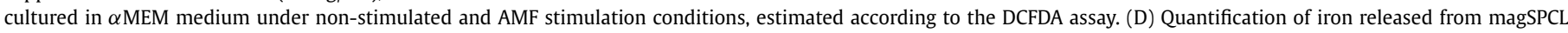

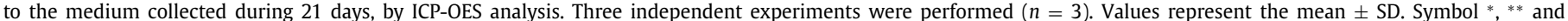
**** denotes study groups with statistical significant difference $p<0.05, p<0.01$ and $p<0.0001$, respectively; a and b letters represent statistically different groups.

labeling effect of the conjugated MNPs with the negatively charged cell membrane. The morphology of the MNPs and MNPs-ActRIIA was analysed by SEM (Fig. 3B) showing the expected round shape of the particles. Also, particle aggregates are diminished on samples upon functionalization with antibody.

FTIR spectrum (Fig. 3C) of the MNPs (blue) exhibits the characteristic peak of carbonyl stretch $\mathrm{C}=\mathrm{O}$ of the carboxylic acids at $1619 \mathrm{~cm}^{-1}$. The MNPs-ActRIIA spectrum (orange) exhibits two bands characteristic of the amide group, amide I band around $1634 \mathrm{~cm}^{-1}$ ( $\mathrm{C}=\mathrm{O}$ stretch) and amide II band ( $\mathrm{N}-\mathrm{H}$ bend) around $1539 \mathrm{~cm}^{-1}$. The replacement of the carboxylic acid group band by two amide group bands strongly suggests that the conjugation between the MNPs and the anti-ActRIIA antibody by EDAC/NHS chemistry was successful.

The efficiency of MNPs conjugation was determined using the BCA protein assay by estimating the amount of anti-ActRIIA conjugated to MNPs (Fig. $3 \mathrm{C}$ ). During the conjugation procedure, the supernatant was collected before and after incubation with the activated MNPs for further protein content measurement. The protein present on the supernatants solutions decreased and a binding efficiency of (37 \pm 13$) \%$ was obtained, which is in good agreement with values found in literature [38].

The labeling of hASCs with MNPs-ActRIIA was confirmed by SEM analysis and Prussian Blue (PB) staining (Fig. 3D) in which it is possible to observe the conjugated MNPs in white (SEM) or blue (PB) targeting the cell membrane after 30 min of labeling period.

\subsection{Assessment of Smad 2/3 phosphorylation in hASCs triggered by Activin A or MNPs-ActRIIA}

The binding of TGF- $\beta$ family ligands to cell membrane receptors, such as ActRIIA, induces phosphorylation and activation of the receptor initiating an intracellular signaling that leads to the phosphorylation of Smad proteins [39]. Thus, the activation of the ActRIIA was indirectly assessed through detection of the downstream phosphorylation of Smad2/3 proteins (Fig. 4A).

The capability of functionalized MNPs to remotely target and activate the ActRIIA receptor via magnetic mechano stimulation was investigated using AMF stimulation (Fig. 4B1). The higher phosphorylation of Smad2/3 in hASCs labelled with MNPs-ActRIIA under AMF stimulation suggested that ActRIIA is a mechanosensitive receptor that can be activated using MNPs-ActRIIA, which was also verified elsewhere, however using a different magnetic device [13]. The actuation of an external AMF causes vibrations of the MNPs-Ab complex enhancing mechano activation of the receptor $[13,18,19]$.

In the inhibition experiments, hASCs were treated with SB431542, an exogenous antagonist against the activin receptor and, consequently, reported to inhibit the TGF- $\beta / \operatorname{Smad} 2 / 3$ signaling $[32,40]$. After confirming the involvement of the antagonist in the inhibition of the receptor, a rescue experiment was performed to insight on the reactivation of the phosphorylation cascade by the ligands. Thus, pre-treated hASCs with SB431542 supplemented medium were incubated with Activin A or MNPs-ActRIIA under 
A

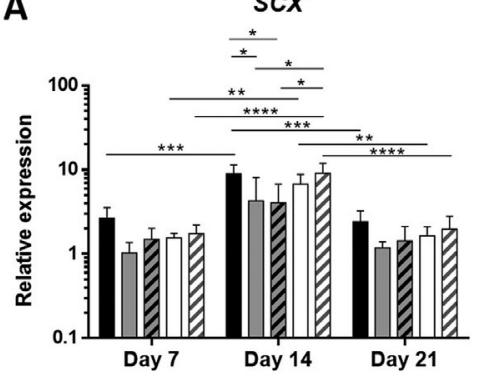

$D C N$

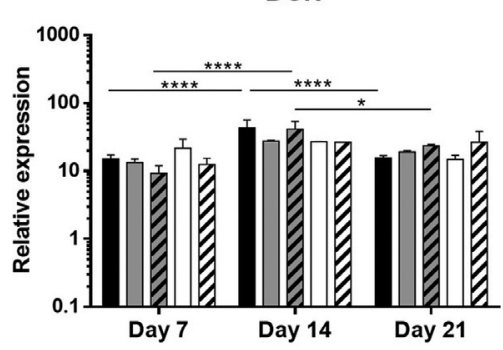

TNMD

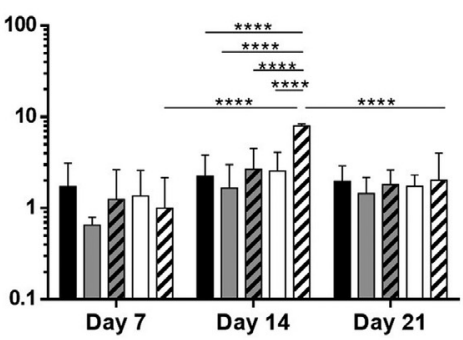

TNC

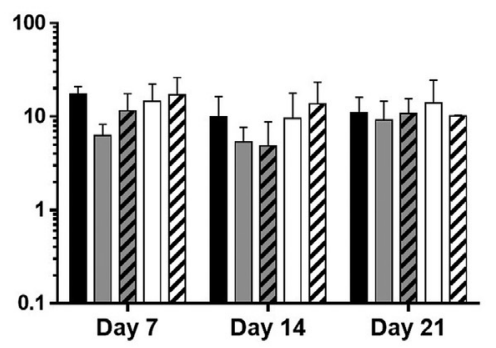

COL1A1

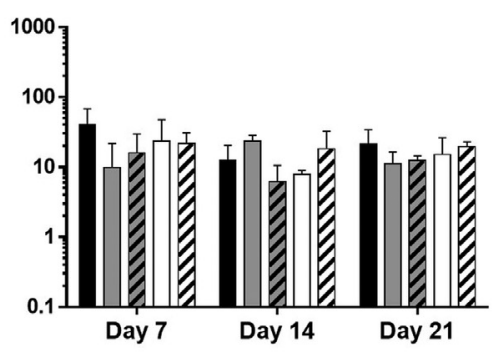

Activin A 20ng/mL hASCs non-stimulated ZI hASCs AMF stimulation $\square$ MNPs-ActRIIA non-stimulated ZZ MNPs-ActRIIA AMF stimulation
B

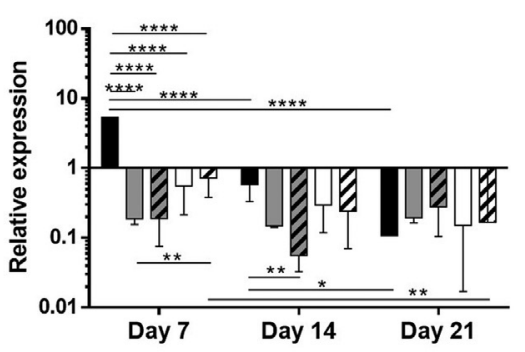

ACAN

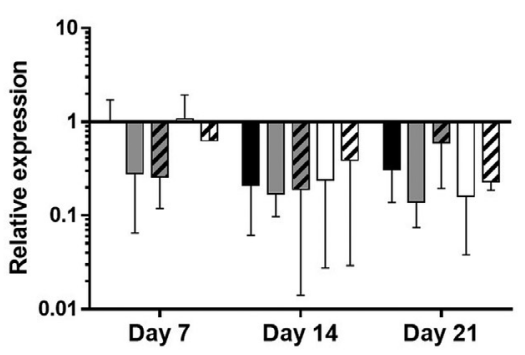

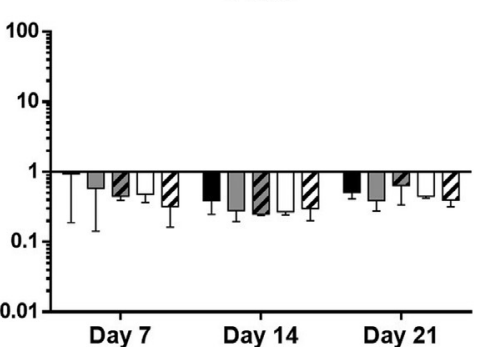

PPAR- $\gamma$

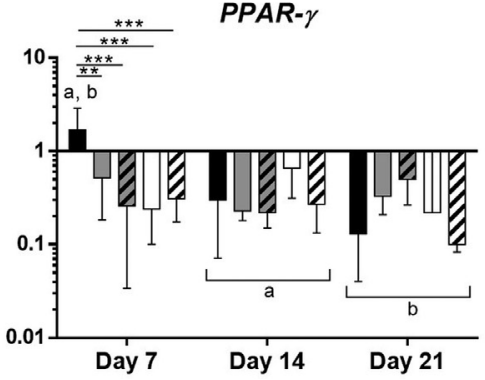

Activin A 20ng/mL hASCs non-stimulated hASCs AMF stimulation MNPs-ActRIIA non-stimulated MNPs-ActRIIA AMF stimulation

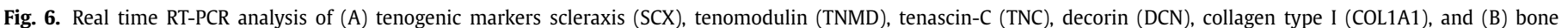

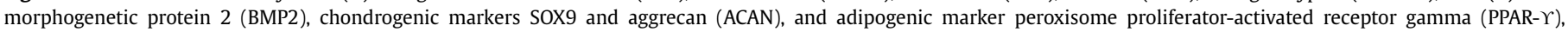

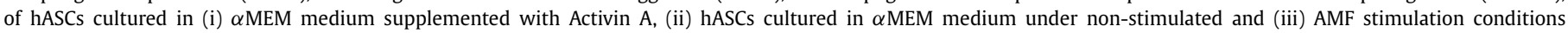

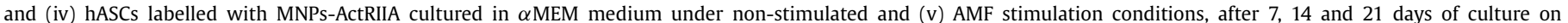

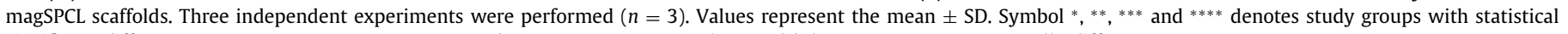
significant difference $p<0.05, p<0.01, p<0.001$ and $p<0.0001$, respectively. a and b letters represent statistically different groups.

non-stimulated or AMF stimulation conditions (Fig. 4B2). The phosphorylation values of cells treated with Activin A or hASCs untreated were significantly different $(p<0.05)$ than the values of all samples that were pre-exposed to the antagonist. Overall, the results showed that hASCs treatment with SB431542 was able to inhibit the receptor and subsequent Smad2/3 pathway which is in agreement with Inman GJ et al. that demonstrated that inhibition of the activin receptors with SB431542 and the subsequent treatment with TGF- $\beta$ and Activin ligands does not rescue pSmad2 in keratinocytes, fibroblasts, and myoblasts cell lines [32].

In magSPCL constructs, there is a higher phosphorylation tendency of the protein complex in hASCs labelled with MNPs-ActRIIA under actuation of AMF ( $p>0.05)$, what may suggest a more efficient activation of the ActRIIA receptor (Fig. 4C). 
Collagen

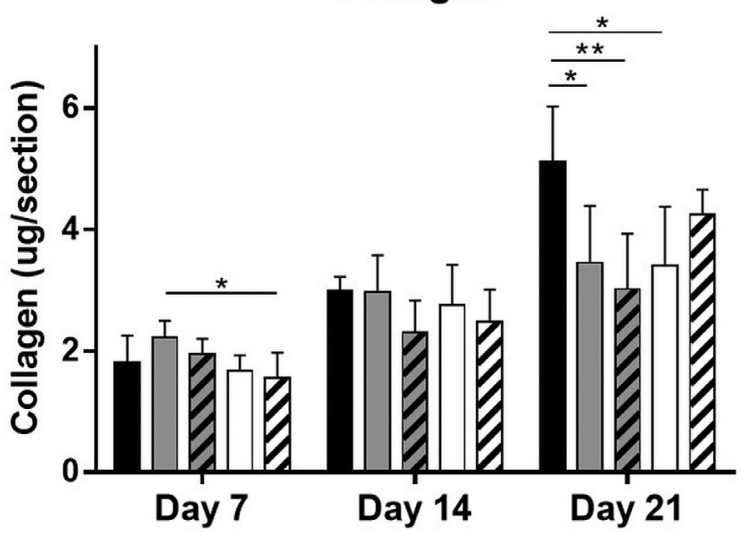

$\square$

Activin A 20ng/mL

hASCs non-stimulated hASCs AMF stimulation
Non-collagenous proteins

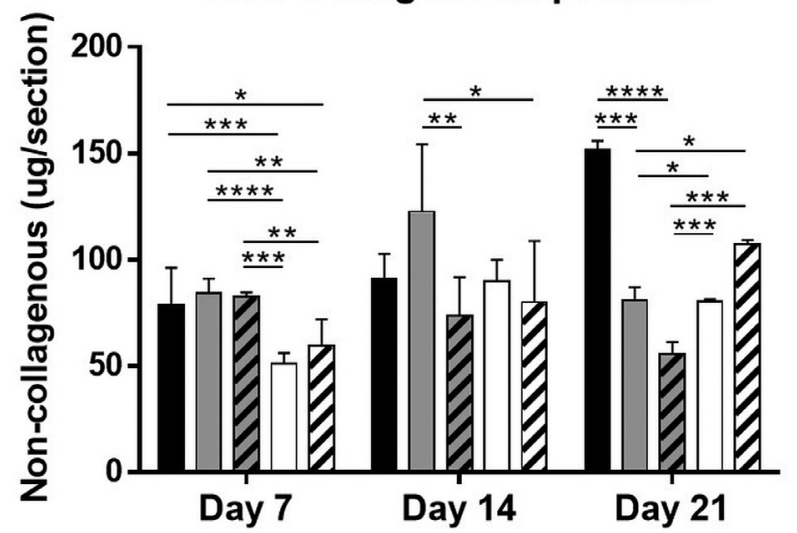

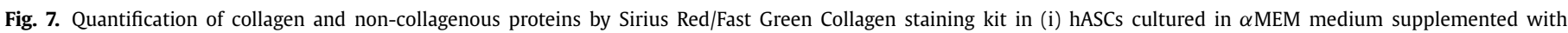

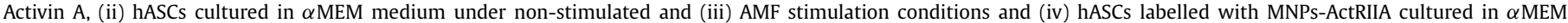

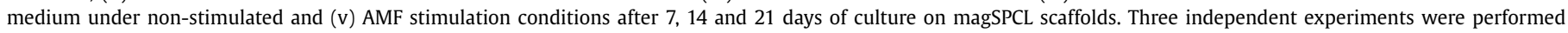

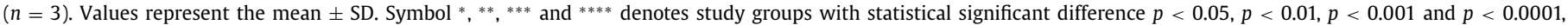
respectively.

The activation of the ActRIIA induces phosphorylation of the Smad2/3 proteins that translocate to the nucleus regulating the expression of target genes [41]. In our study, the immunolocation of pSmad2/3 is more easily observed in the nucleus on hASCs labelled with MNPs-ActRIIA seeded on magSPCL scaffolds under AMF stimulation conditions, which also confirms that the actuation of AMF on MNPs-ActRIIA enhances the activation of the receptor via magnetic mechano actuation (Fig. 4D1, D2). These findings suggest that the actuation of a magnetic field on a magnetic scaffold induces local substrate deflections creating transient physical forces sensed by the cultured cells in close proximity to the nanoparticles which promotes mechanotransduction mechanisms $[5,11]$, possibly driving differentiation of stem cells. This also supports the obtained very weak pSmad2/3 signals in the nuclei of unlabeled hASCs laden on magnetic scaffolds. On the other hand, the MNPs tagged hASCs stimulated by an AMF will sense translational forces directed along the magnetic field inducing the motion of MNPs attached via membrane receptors, ultimately activating mechanotransduction processes [7]. It has been also reported that less than $0.2 \mathrm{pN}$ was required to activate the TREK- 1 channel [6], and the estimated magnetically induced mechanical force imparted on cells grown on alginate magnetic scaffolds was reported in the order of $1 \mathrm{pN}$ [5] to induce mechanoresponsive effects at the cellular level.

Although the precise constructs deformation upon magnetic stimulation was not investigated in the present work, we hypothesize that the total forces applied in our constructs as a resulting combination of actuable magSPCL and MNPs-ActRIIA labelled hASCs leads to a more pronounced tenogenic response. However, forthcoming work is needed to overcome this limitation.

\section{4. hASCS metabolic activity, proliferation, oxidative stress, and iron release}

The metabolic activity and cell proliferation of hASCs were assessed by MTS assay and dsDNA quantification, respectively (Fig. 5A and Fig. 5B). The results showed an increase in both metabolic activity and cell proliferation after 21 days in culture suggesting that the external AMF applied to the 3D constructs did not affect negatively the cell behavior.

Reactive oxygen species (ROS) regulate cell homeostasis and may accumulate in response to environmental stress disrupting normal cellular processes [42]. The role of the magnetic field in oxidative stress is controversial, however, ROS assay was performed to study the effect of AMF stimulation on hASCs response (Fig. 5C). Our results showed no difference between samples under AMF stimulation and non-stimulated conditions, suggesting that environmental stress produced by the actuation of the AMF is not deleterious for hASCs.

Furthermore, in order to evaluate the possible release of MNPs from the magnetic scaffolds, the medium collected during the cell culture assay was analysed for elemental iron by ICP- OES (Fig. 5D). All values of iron concentration were normalized in relation to $\alpha$-MEM medium (supplemented with $10 \%$ FBS and $1 \%$ A/A) that presents an iron concentration of $290 \pm 0.01 \mu \mathrm{g} \mathrm{L}^{-1}$. The overall release of iron from magSPCL scaffolds (without cells) into the culture medium was higher in the presence of an external AMF in comparison with non-stimulated conditions. The actuation of a magnetic field on the 3D constructs is expected to cause minor vibrations of the MNPs incorporated in the scaffold structure [5] and, consequently, this might promote to some extent, particles release from the fibers, which explains the increase of iron release under AMF stimulation. This also corroborates with previously reported data of our group [12]. Moreover, the release of iron to the medium was higher in cell-free samples in comparison with cells laden scaffolds, at all time-points. This suggests that the iron contribution to the culture medium derives from the scaffold's embedded MNPs and not from MNPs-ActRIIA labelled hASCs.

\subsection{Gene expression}

The effect of an externally applied AMF was investigated over the 21 days culture period in the tenogenic commitment of hASCs. Real time reverse transcription polymerase chain reaction (RT-PCR) was performed at day 7,14 and 21, particularly focusing on a panel of tendon related markers including SCX, TNMD, DCN, TNC, and 
A TNMD
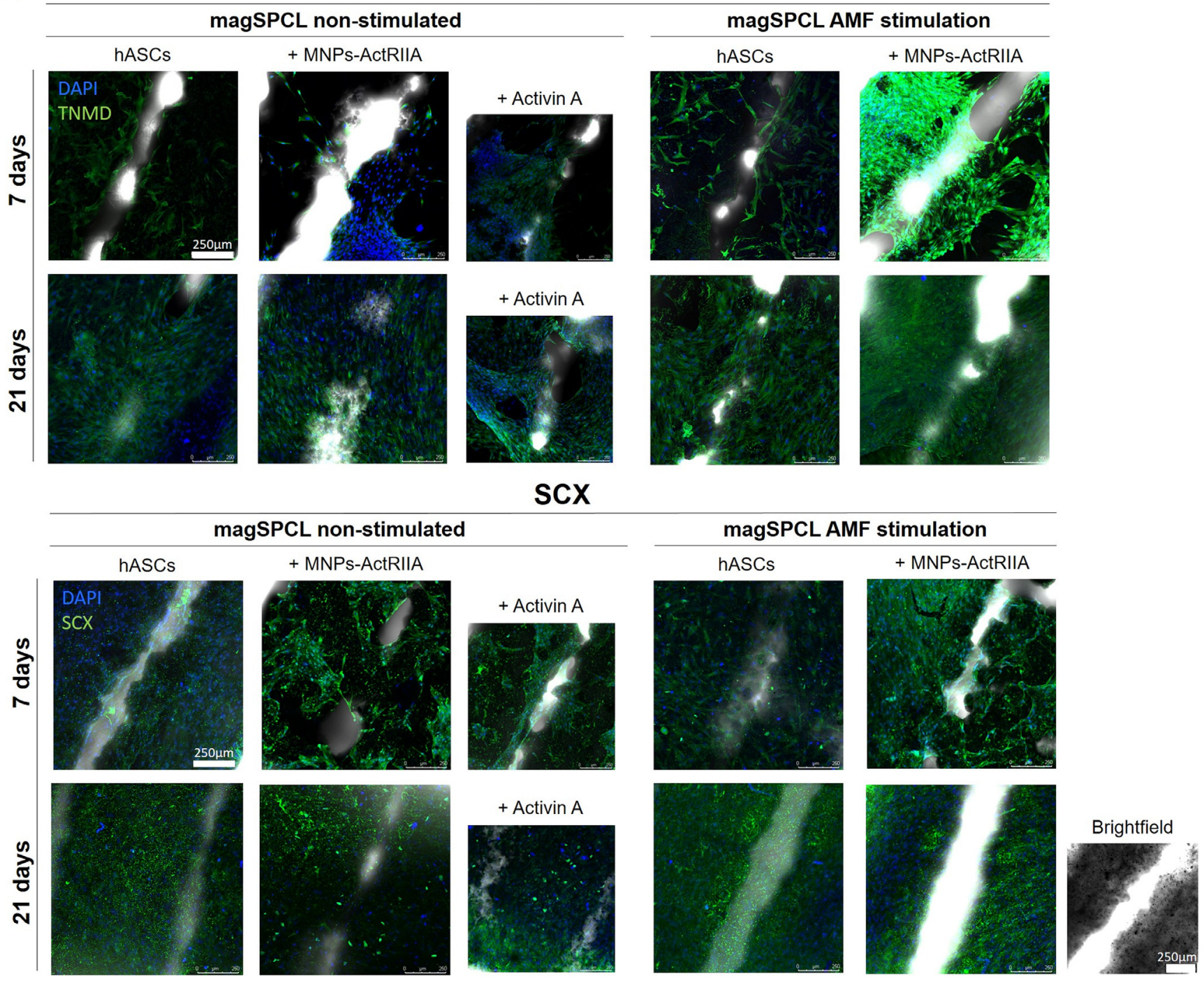

B

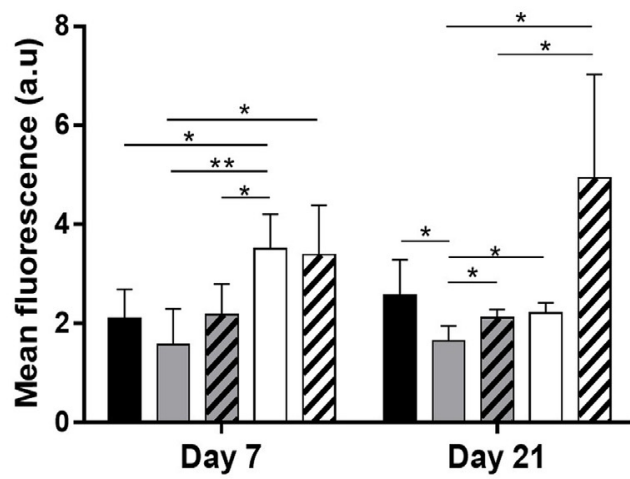

$\operatorname{scX}$

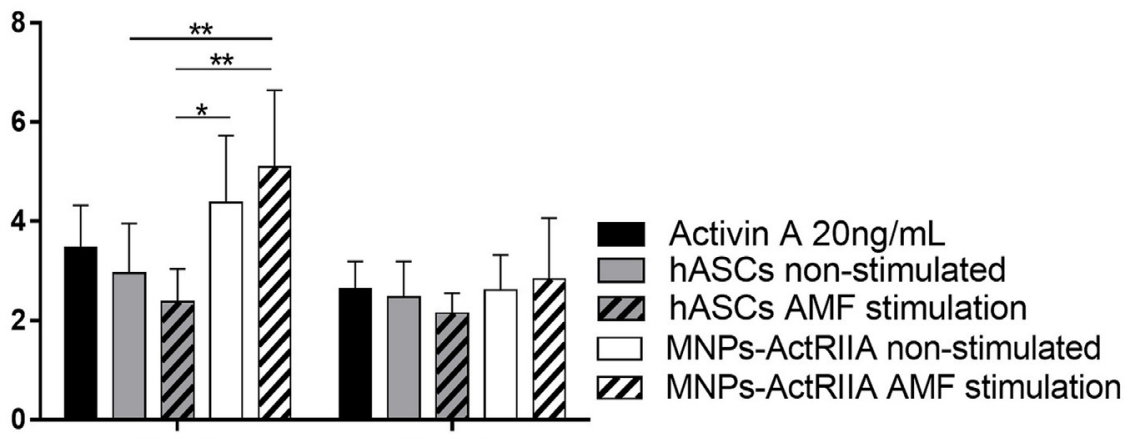

Day 7
Day 21

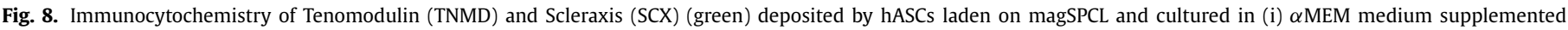

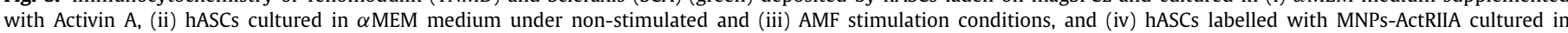

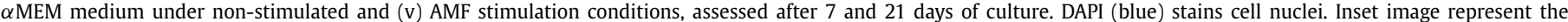

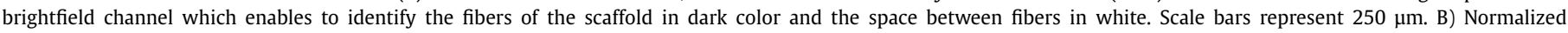

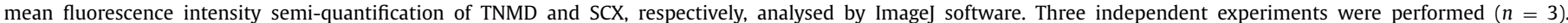

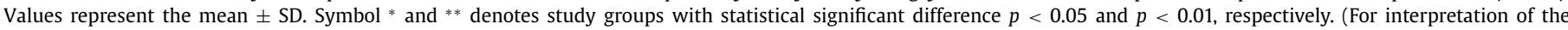
references to color in this figure legend, the reader is referred to the web version of this article.) 
COL1A1 (Fig. 6A). The expression of $S C X$ and TNMD was higher in AMF stimulation conditions at 14 days $(p<0.05)$. Magnetic field actuating on hASCs laden on magnetic fibrous scaffolds was shown to activate the transcriptional factors YAP and TAZ [11], which upon activation are translocated to the nucleus, promoting stem cell differentiation. It was verified by Tomás AR et al. an increase in nuclear YAP/TAZ, indicative of tension of the cytoskeleton which was likely caused by its adjustment to the external physical stimulus $[11,43]$. This mechanotransduction mechanism was suggested to boost hASCs tenogenic differentiation [11], further supporting our results. Similarly, in our study, the more effective activation of TGF- $\beta / \operatorname{Smad} 2 / 3$ mechanism under AMF actuation, triggered the expression of target genes. After 21 days in culture, the expression of these genes was higher on hASCs labelled with MNPs-ActRIIA seeded on magSPCL scaffolds under AMF stimulation. Beyond magnetically actuated magSPCL potential per se in mediating external stimulus and cellular responses, these results also suggest that directly targeting the ActRIIA receptor by magnetic mechano activation, enhanced the upregulation of tendon related genes.

On the other hand, the overall expression of non-tendon related markers tended to be downregulated in all conditions and timepoints (Fig. 6B). Particularly from day 7 to 21, the expression of BMP2, an osteogenic marker [44], significantly decreased in AMF stimulated hASCs-MNPs-ActRIIA cultured in magSPCL $(p<0.01)$. The chondrogenic markers SOX9 [45] and ACAN expressions were also downregulated throughout the time in all conditions. Moreover, and despite of the adipose tissue source of stem cells used in this study, the expression of the adipogenic-related gene PPAR$r$ was downregulated throughout the time course of the experiment. PPAR- $Y$ expression after 14 and 21 days was lower in AMF stimulated hASCs labelled with MNPs-ActRIIA seeded on magSPCL scaffolds (Fig. 6B). Taken together, these results suggest that the tenogenic commitment of hASCs was enhanced by the synergy between remote magnetic actuation via MNPs-ActRIIA and via 3D magSPCL scaffolds, in regulating the tenogenic phenotype of hASCs while decreasing the expression of genes related to other lineages.

\subsection{Extracellular matrix formation (ECM)}

The amount of collagen and non-collagenous proteins produced by hASCs labelled with MNPs-ActRIIA seeded on magSPCL scaffolds under AMF stimulation tended to increase from day 7 to 21 days in culture (Fig. 7). At day 7, there is greater collagen production in hASCs constructs compared to all other groups. Moreover, at day 21 there is no difference in collagen production between nonstimulated and AMF stimulated groups. Contrariwise, it is possible to observe that the production of non-collagenous proteins after 21 days by hASCs labelled with MNPs-ActRIIA seeded on magSPCL scaffolds under AMF stimulation is significantly higher compared to non-labelled hASCs either AMF stimulated $(p<0.001)$ or not $(p<0.05)$. Interestingly, MNPs-ActRIIA labelled cells produce more non-collagenous proteins $(p<0.001)$ in static scaffolds than plain cells AMF stimulated, suggesting a key role in triggered ActRIIA cell receptors.

The tenogenic commitment of hASCs was also evaluated by studying the deposition of two of the most widely accepted tenogenic markers, SCX and TNMD [46], by immunocytochemistry (Fig. 8A) after 7 and 21 days. At both time-points, TNMD deposition tended to enhance in hASCs labelled with MNPs-ActRIIA laden on magSPCL scaffolds under AMF stimulation (Fig. 8B). On the other hand, the expression of SCX was significantly higher on hASCs labelled with MNPs-ActRIIA in comparison with the conditions comprising unlabeled cells at day 7 (Fig. 8B), and no differences were observed at day 21 for SCX. Comparing MNPs-ActRIIA tagged hASCs under non-stimulated or AMF stimulation conditions, the actuation of a magnetic field increased SCX production at the protein level. A higher level of SCX was observed in the condition with MNPs-ActRIIA under AMF stimulation at both time-points. However, we observe a decrease tendency from 7 to 21 days. The transcription factor SCX is mainly expressed during early tendon development thus regulating tissue formation $[2,46]$. Also, the fact that the production of SCX at day 7 by hASCs labelled with MNPsActRIIA seeded on magSPCL scaffolds under AMF stimulation is higher, and the increased TNMD deposition at day 21 in the same condition is in agreement with the role of SCX as a transcriptional inducer of TNMD $[22,47]$.

\section{Conclusions}

The technology of remote activation of mechanotransduction via magnetic nanoparticles (MNPs) has been broadly employed to trigger different cellular receptors or ion channels. In the present study, we have successfully used this approach in a 3D environment comprising magnetic scaffolds laden with MNPs-ActRIIA tagged hASCs and exposed to the actuation of an externally applied alternating magnetic field. Remote magnetic actuation of MNPsActRIIA complex tagging the mechanosensitive ActRIIA receptor of hASCs was able to activate the TGF- $\beta$ signaling pathway via Smad2/3 cascade both in 2D and 3D cultures, as shown by phosphorylation of Smad2/3 proteins and their nuclear co-localization. Also, the synergistic effect of the magnetically responsive scaffolds and MNPs-ActRIIA tagged hASCs boosted the commitment of hASCs into the tenogenic lineage.

The potential application of this engineered magneticallyresponsive system in vivo may provide a new platform to commit hASCs towards tenogenesis by modulating the TGF- $\beta / \operatorname{Smad} 2 / 3$ signaling pathway involved in tendon repair and improving the potential of TE strategies in the effective regeneration of functional tendon tissue.

\section{Disclosures}

Authors have nothing to disclosure.

\section{Declaration of Competing Interest}

The authors declare that they have no known competing financial interests or personal relationships that could have appeared to influence the work reported in this paper.

\section{Acknowledgements}

Authors acknowledge the project "Accelerating tissue engineering and personalized medicine discoveries by the integration of key enabling nanotechnologies, marine-derived biomaterials and stem cells", supported by Norte Portugal Regional Operational Programme (NORTE 2020), under the PORTUGAL 2020 Partnership Agreement, through the European Regional Development Fund (ERDF), and the FCT Project MagTT PTDC/CTM-CTM/29930/2017 (POCI-01-0145-FEDER-29930).

Authors acknowledge the HORIZON 2020 for the Achilles Twinning Project No. 810850. Authors also thank the European Research Council COG MagTendon No. 772817 and the ADG DYNACEUTICS No. 789119.

Prof. Bernardo Almeida from Physics Department, University of Minho, is also acknowledged for assisting in the magnetic system assembling.

Authors also acknowledge the INL - International Iberian Nanotechnology Laboratory (Braga, Portugal) for the magnetization analysis. 


\section{Supplementary materials}

Supplementary material associated with this article can be found, in the online version, at doi:10.1016/j.actbio.2020.07.009.

\section{References}

[1] J.G. Snedeker, J. Foolen, Tendon injury and repair - A perspective on the basic mechanisms of tendon disease and future clinical therapy, Acta Biomater. 63 (2017) 18-36.

[2] G. Nourissat, F. Berenbaum, D. Duprez, Tendon injury: from biology to tendon repair, Nat. Rev. Rheumatol. 11 (4) (2015) 223-233.

[3] X. Zhao, S. Jiang, S. Liu, S. Chen, Z.Y.W. Lin, G. Pan, F. He, F. Li, C. Fan, W. Cui, Optimization of intrinsic and extrinsic tendon healing through controllable water-soluble mitomycin-C release from electrospun fibers by mediating adhesion-related gene expression, J. Biomater. 61 (2015) 61-74.

[4] M.T. Rodrigues, R.L. Reis, M.E. Gomes, Engineering tendon and ligament tissues: present developments towards successful clinical products, J. Tissue Eng. Regen. Med. 7 (9) (2013) 673-686.

[5] Y. Sapir-Lekhovitser, M.Y. Rotenberg, J. Jopp, G. Friedman, B. Polyak, S. Cohen, Magnetically actuated tissue engineered scaffold: insights into mechanism of physical stimulation, Nanoscale 8 (6) (2016) 3386-3399.

[6] S. Hughes, S. McBain, J. Dobson, A.J. El Haj, Selective activation of mechanosensitive ion channels using magnetic particles, J. R. Soc. Interface 5 (25) (2008) 855-863.

[7] J. Dobson, S.H. Cartmell, A. Keramane, A.J. El Haj, Principles and design of a novel magnetic force mechanical conditioning Bioreactor for tissue engineering, stem cell conditioning, and dynamic in vitro screening, IEEE Trans. Nanobiosci. 5 (3) (2006) 173-177.

[8] J. Meng, B. Xiao, Y. Zhang, J. Liu, H.D. Xue, J. Lei, H. Kong, Y.G. Huang, Z.Y. Jin, N. Gu, H.Y. Xu, Super-paramagnetic responsive nanofibrous scaffolds under static magnetic field enhance osteogenesis for bone repair in vivo, Sci. Rep. 3 (2013) 1-7.

[9] Y. Sapir, B. Polyak, S. Cohen, Cardiac tissue engineering in magnetically actuated scaffolds, Nanotechnology 25 (1) (2014) 014009.

[10] Y. Sapir, S. Cohen, G. Friedman, B. Polyak, The promotion of in vitro vessel-like organization of endothelial cells in magnetically responsive alginate scaffolds, Biomaterials 33 (16) (2012) 4100-4109.

[11] A.R. Tomas, A.I. Goncalves, E. Paz, P. Freitas, R.M.A. Domingues, M.E. Gomes, Magneto-mechanical actuation of magnetic responsive fibrous scaffolds boosts tenogenesis of human adipose stem cells, Nanoscale 11 (39) (2019) $18255-18271$.

[12] A.I. Goncalves, M.T. Rodrigues, P.P. Carvalho, M. Banobre-Lopez, E. Paz, P. Freitas, M.E. Gomes, Exploring the potential of starch/polycaprolactone aligned magnetic responsive scaffolds for tendon regeneration, Adv. Healthc. Mater. 5 (2) (2016) 213-222.

[13] A.I. Goncalves, M. Rotherham, H. Markides, M.T. Rodrigues, R.L. Reis, M.E. Gomes, A.J. El Haj, Triggering the activation of Activin A type II receptor in human adipose stem cells towards tenogenic commitment using mechanomagnetic stimulation, Nanomed. Nanotechnol. Biol. Med. 14 (4) (2018) 1149-1159.

[14] A.I. Gonçalves, M.T. Rodrigues, M.E. Gomes, Tissue-engineered magnetic cell sheet patches for advanced strategies in tendon regeneration, Acta Biomater. 63 (2017) 110-122.

[15] A. Vinhas, M.T. Rodrigues, A.I. Gonçalves, R.L. Reis, M.E. Gomes, Pulsed electromagnetic field modulates tendon cells response in IL- $1 \beta$; conditioned environment, J. Orthop. Res. 38 (1) (2019) 160-172.

[16] L. Santos, M. Silva, A.I. Goncalves, T. Pesqueira, M.T. Rodrigues, M.E. Gomes, In vitro and in vivo assessment of magnetically actuated biomaterials and prospects in tendon healing, Nanomedicine 11 (9) (2016) 1107-1122.

[17] J.R. Henstock, M. Rotherham, A.J. El Haj, Magnetic ion channel activation of TREK1 in human mesenchymal stem cells using nanoparticles promotes osteogenesis in surrounding cells, J. Tissue Eng. 9 (2018) 1-10.

[18] M. Rotherham, A.J. El Haj, Remote activation of the Wnt/beta-catenin signalling pathway using functionalised magnetic particles, PLoS One 10 (3) (2015) e0121761.

[19] B. Hu, J. Dobson, A.J. El Haj, Control of smooth muscle alpha-actin (SMA) up-regulation in HBMSCs using remote magnetic particle mechano-activation, Nanomed.Nanotechnol. 10 (1) (2014) 45-55.

[20] J.D. Humphrey, E.R. Dufresne, M.A. Schwartz, Mechanotransduction and extracellular matrix homeostasis, Nat. Rev. Mol. Cell Biol. 15 (12) (2014) 802-812.

[21] E. Havis, M.A. Bonnin, I. Olivera-Martinez, N. Nazaret, M. Ruggiu, J. Weibel, C. Durand, M.J. Guerquin, C. Bonod-Bidaud, F. Ruggiero, R. Schweitzer, D. Duprez, Transcriptomic analysis of mouse limb tendon cells during development, Development 141 (19) (2014) 3683-3696.
[22] J. Qi, J.M. Dmochowski, A.N. Banes, M. Tsuzaki, D. Bynum, M. Patterson, A. Creighton, S. Gomez, K. Tech, A. Cederlund, Differential expression and cellular localization of novel isoforms of the tendon biomarker tenomodulin, J. Appl. Physiol. 113 (6) (2012) 861-871.

[23] S. Dex, P. Alberton, L. Willkomm, T. Söllradl, S. Bago, S. Milz, M. Shakibaei, A. Ignatius, W. Bloch, H. Clausen-Schaumann, Tenomodulin is required for tendon endurance running and collagen I fibril adaptation to mechanical load, J. EBio. Med. 20 (2017) 240-254.

[24] S. Dex, D.S. Lin, C. Shukunami, D. Docheva, TENOgenic MODULating INsider factor: systematic assessment on the functions of tenomodulin gene, Gene 587 (1) (2016) 1-17.

[25] T. Maeda, T. Sakabe, A. Sunaga, K. Sakai, A.L. Rivera, D.R. Keene, T. Sasaki, E. Stavnezer, J. Iannotti, R. Schweitzer, D. Ilic, H. Baskaran, T. Sakai, Conversion of mechanical force into TGF-beta-mediated biochemical signals, Curr. Biol. 21 (11) (2011) 933-941.

[26] M.E. Gomes, H.S. Azevedo, A. Moreira, V. Ellä, M. Kellomäki, R. Reis, Starch-poly ( $\varepsilon$-caprolactone) and starch-poly (lactic acid) fibre-mesh scaffolds for bone tissue engineering applications: structure, mechanical properties and degradation behaviour, J. Tissue Eng. Regen. Med. 2 (5) (2008) 243-252.

[27] T. Rada, R. Reis, M.E. Gomes, R. Medicine, Novel method for the isolation of adipose stem cells (ASCs), J. Tissue Eng. 3 (2) (2009) 158-159.

[28] P.P. Carvalho, X. Wu, G. Yu, I.R. Dias, M.E. Gomes, R.L. Reis, J.M. Gimble, The effect of storage time on adipose-derived stem cell recovery from human lipoaspirates, J. Cells Tissues Organs 194 (6) (2011) 494-500.

[29] P.P. Carvalho, X. Wu, G. Yu, M. Dietrich, I.R. Dias, M.E. Gomes, R.L. Reis, J.M. Gimble, Use of animal protein-free products for passaging adherent human adipose-derived stromal/stem cells, Cytotherapy 13 (5) (2011) 594-597.

[30] Y. Sapir, S. Cohen, G. Friedman, B. Polyak, The promotion of in vitro vessel-like organization of endothelial cells in magnetically responsive alginate scaffolds, J Biomaterials 33 (16) (2012) 4100-4109.

[31] L. Attisano, J.L. Wrana, E. Montalvo, J. Massague, Activation of signalling by the activin receptor complex, Mol. Cell. Biol. 16 (3) (1996) 1066-1073.

[32] G.J. Inman, F.J. Nicolas, J.F. Callahan, J.D. Harling, L.M. Gaster, A.D. Reith, N.J. Laping, C.S. Hill, SB-431542 is a potent and specific inhibitor of transforming growth factor-beta superfamily type I activin receptor-like kinase (ALK) receptors ALK4, ALK5, and ALK7, Mol. Pharmacol. 62 (1) (2002) 65-74.

[33] M. Sezgin, B. Sankur, Survey over image thresholding techniques and quantitative performance evaluation, J. Electron. Imaging 13 (1) (2004).

[34] L.-.K. Huang, M.-J.J. Wang, Image thresholding by minimizing the measures of fuzziness, Pattern Recognit. 28 (1) (1995) 41-51.

[35] S. Hao, J. Meng, Y. Zhang, J. Liu, X. Nie, F. Wu, Y. Yang, C. Wang, N. Gu, H. Xu, Macrophage phenotypic mechanomodulation of enhancing bone regeneration by superparamagnetic scaffold upon magnetization, J. Biomater. 140 (2017) $16-25$.

[36] P.P. Carvalho, M.T. Rodrigues, M.E. Gomes, R.L. Reis, John Wiley \& Sons, 2016, pp. 244-257.

[37] A.M. Martins, Q.P. Pham, P.B. Malafaya, R.A. Sousa, M.E. Gomes, R.M. Raphael, F.K. Kasper, R.L. Reis, A.G. Mikos, The role of lipase and $\alpha$-amylase in the degradation of starch/poly ( $\varepsilon$-caprolactone) fiber meshes and the osteogenic differentiation of cultured marrow stromal cells, J. Tissue Eng. Part A 15 (2) (2008) 295-305.

[38] M. Rotherham, J.R. Henstock, O. Qutachi, A.J. El Haj, Remote regulation of magnetic particle targeted Wnt signaling for bone tissue engineering, Nanomedicine: NBM 14 (1) (2018) 173-184.

[39] L.M. Wakefield, C.S. Hill, Beyond TGF $\beta$ : roles of other TGF $\beta$ superfamily members in cancer, Nat. Rev. Cancer 13 (5) (2013) 328-341.

[40] C.A. Harrison, P.C. Gray, W.W. Vale, D.M. Robertson, Metabolism, Antagonists of activin signaling: mechanisms and potential biological applications, J. Trends Endocrinology 16 (2) (2005) 73-78.

[41] A. Subramanian, T.F. Schilling, Tendon development and musculoskeletal assembly: emerging roles for the extracellular matrix, J Development 142 (24) (2015) 4191-4204.

[42] H. Wang, X. Zhang, Magnetic fields and reactive oxygen species, Int. J. Mol. Sci. 18 (10) (2017) 2175.

[43] T.P. Driscoll, B.D. Cosgrove, S.-.J. Heo, Z.E. Shurden, R.L. Mauck, Cytoskeletal to nuclear strain transfer regulates YAP signaling in mesenchymal stem cells, J Biophys. J. 108 (12) (2015) 2783-2793.

[44] V. Rosen, BMP2 signaling in bone development and repair, Cytokine Growth Factor Rev. 20 (5-6) (2009) 475-480.

[45] V. Lefebvre, M. Angelozzi, A. Haseeb, SOX9 in cartilage development and disease, Curr. Opin. Cell Biol. 61 (2019) 39-47.

[46] L. Gaut, D. Duprez, Tendon development and diseases, Wiley Interdiscip. Rev. Dev. Biol. 5 (1) (2016) 5-23.

[47] C. Shukunami, A. Takimoto, Y. Nishizaki, Y. Yoshimoto, S. Tanaka, S. Miura, H. Watanabe, T. Sakuma, T. Yamamoto, G. Kondoh, Scleraxis is a transcriptional activator that regulates the expression of Tenomodulin, a marker of mature tenocytes and ligamentocytes, J. Sci. Rep. 8 (1) (2018) 3155. 